\title{
Cortical Interneurons Require Jnkl to Enter and Navigate the Developing Cerebral Cortex
}

\author{
Abigail K. Myers, ${ }^{1,2,3}$ Daniel W. Meechan, ${ }^{4}$ Danielle R. Adney, ${ }^{1,3}$ and Eric S. Tucker ${ }^{1,3}$ \\ ${ }^{1}$ Department of Neurobiology and Anatomy, ${ }^{2}$ Neuroscience Graduate Program, and ${ }^{3}$ Center for Neuroscience, West Virginia University School of Medicine, \\ Morgantown, West Virginia 26506, and ${ }^{4}$ Department of Pharmacology and Physiology, The George Washington University Medical Center, Washington, \\ DC 20037
}

\begin{abstract}
Proper assembly of cortical circuitry relies on the correct migration of cortical interneurons from their place of birth in the ganglionic eminences to their place of terminal differentiation in the cerebral cortex. Although molecular mechanisms mediating cortical interneuron migration have been well studied, intracellular signals directing their migration are largely unknown. Here we illustrate a novel and essential role for c-Jun N-terminal kinase (JNK) signaling in guiding the pioneering population of cortical interneurons into the mouse cerebral cortex. Migrating cortical interneurons express Jnk proteins at the entrance to the cortical rudiment and have enriched expression of $J n k 1$ relative to noninterneuronal cortical cells. Pharmacological blockade of JNK signaling in ex vivo slice cultures resulted in dose-dependent and highly specific disruption of interneuron migration into the nascent cortex. Time-lapse imaging revealed that JNK-inhibited cortical interneurons advanced slowly and assumed aberrant migratory trajectories while traversing the cortical entry zone. In vivo analyses of JNK-deficient embryos supported our ex vivo pharmacological data. Deficits in interneuron migration were observed in Jnk1 but not Jnk2 single nulls, and those migratory deficits were further exacerbated when homozygous loss of Jnk1 was combined with heterozygous reduction of $J n k 2$. Finally, genetic ablation of $J n k 1$ and $J n k 2$ from cortical interneurons significantly perturbed migration in vivo, but not in vitro, suggesting JNK activity functions to direct their guidance rather than enhance their motility. These data suggest JNK signaling, predominantly mediated by interneuron expressed Jnk1, is required for guiding migration of cortical interneurons into and within the developing cerebral cortex.
\end{abstract}

Key words: development; forebrain; GABAergic interneuron; intracellular signaling; neuronal migration; psychiatric disorder

\section{Introduction}

Cortical interneurons play vital roles regulating neurotransmission in the cerebral cortex and their dysfunction is implicated in severe brain disorders including epilepsy and schizophrenia. Mechanisms contributing to the pathological vulnerability of cortical interneurons are unclear, but they may arise during embryonic development when interneurons embark on long distance tangential migration from the medial and caudal ganglionic eminences to the overlying cerebral cortex (Anderson et al., 1997; Lavdas et al., 1999; Sussel et al., 1999; Wichterle et al., 2001; Nery

Received Nov. 5, 2013; revised March 29, 2014; accepted April 27, 2014.

Author contributions: A.K.M. and E.S.T. designed research;A.K.M., D.W.M., D.R.A., and E.S.T. performed research; A.K.M. and E.S.T. analyzed data; A.K.M. and E.S.T. wrote the paper.

This work was supported by institutional start-up funds from West Virginia University (WVU) to E.T. and core resource support from National Institutes of Health (NIH) Grant P30 GM103503 to the WVU Center for Neuroscience. NIH Grants R01 HD029178 and R01 HD042182 to Anthony-Samuel LaMantia (A.-S.L.) supported initial work for the project while E.S.T. was a postdoctoral fellow with A.-S.L. at University of North Carolina Chapel Hill, as well as data generated in collaboration with D.W.M. at George Washington University. We thank Breeana Baker and Catherine Smith for providing excellent technical support and laboratory management; Danielle Doll, Steven Brooks, John Snow, and Catherine Smith for assistance in data analyses; and Drs. Troy Ghashghaei, Aric Agmon, and Pete Mathers for helpful comments on this manuscript.

The authors declare no competing financial interests.

Correspondence should be addressed to Dr. Eric S. Tucker, Department of Neurobiology and Anatomy, Robert C. Byrd Health Sciences Center, 4052 Health Sciences North, Morgantown, WV 26506-9128. E-mail: etucker@hsc.wvu.edu.

DOI:10.1523/JNEUROSCI.4695-13.2014

Copyright $\odot 2014$ the authors $\quad 0270-6474 / 14 / 347787-14 \$ 15.00 / 0$ et al., 2002; Miyoshi et al., 2010). For this reason, molecular mechanisms guiding cortical interneuron migration have been extensively studied (for review, see Faux et al., 2012; Marín, 2013). Many ligands and receptors mediating cortical interneuron dispersion from the ventral forebrain (Powell et al., 2001), motility (Polleux et al., 2002; Pozas and Ibáñez, 2005), chemorepulsion from the striatum ( Marín et al., 2001), chemoattraction to the cerebral cortex (Yau et al., 2003; Flames et al., 2004), and formation and maintenance of migratory streams have been identified (Tiveron et al., 2006; Li et al., 2008; López-Bendito et al., 2008; Sánchez-Alcañiz et al., 2011; Wang et al., 2011). However, intracellular signals controlling cortical interneuron migration, particularly those directing migration at critical positions along their trajectories, are largely unknown.

The c-Jun N-terminal protein kinase (JNK) pathway plays obligate roles in mammalian forebrain development (Kuan et al., 1999), and moreover, genetic disruption of JNK function leads to cognitive disorders in humans (Kunde et al., 2013). JNKs are members of the mitogen-activated protein kinase (MAPK) signaling pathway, and are encoded by three related genes in mammals: Jnk1 (Mapk8), Jnk2 (Mapk9), and Jnk3 (Mapk10). All three Jnk genes are expressed in the developing mouse brain, but only combinatorial deletion of $J n k 1$ and $J n k 2$ results in embryonic lethality and profound alterations in neuronal survival and programmed cell death (Kuan et al., 1999; Sabapathy et al., 1999). 
Genetic deletions of Jnk1 or upstream Jnk kinases results in aberrant radial migration of cortical projection neurons (Hirai et al., 2006; Wang et al., 2007; Westerlund et al., 2011; Yamasaki et al., 2011), strongly implicating JNK signaling as a major regulator of neuronal migration in the developing forebrain.

In the current study, we use a combination of ex vivo and in vivo approaches to demonstrate that cortical interneurons have a cell-intrinsic requirement for JNK signaling-largely mediated by the activity of Jnk1-to enter and successfully navigate the developing cerebral cortex. JNK-inhibited cortical interneurons dramatically slow their advancement and take inappropriate trajectories at the entrance to the cortical rudiment. Similarly, cortical interneurons in Jnk1- and Jnk1/2-deficent embryos exhibit significant delays in their cortical migration. Conditional ablation of $J n k 1$ and Jnk2 from cortical interneurons delays cortical entry, disrupts migratory streams, and perturbs interneuron morphology in vivo, but has no effect in vitro, suggesting JNK signaling only regulates migration of cortical interneurons in the presence of cortical guidance cues. These findings implicate the JNK pathway, and Jnk1 in particular, as a key molecular node in the intracellular regulation of cortical interneuron migration in vivo.

\section{Materials and Methods}

Animals. Animals were housed and cared for by the Office of Laboratory Animal Resources at West Virginia University. Timed-pregnant dams (day of vaginal plug $=$ embryonic day 0.5 ) were killed by rapid cervical dislocation and mouse embryos were immediately harvested for tissue culture, gene expression, or histological analyses. The following mouse strains were acquired and maintained on a C57BL/6J (The Jackson Laboratory) background: Dlx5/6 Cre-IRES-EGFP (Dlx5/6-CIE; Stenman et al., 2003), Mapk $8^{\text {tmlRjd }}$ floxed (Jnk1 $1^{f l f l}$; Das et al., 2007; kindly provided by Dr. Roger Davis), Mapk $8^{\text {tmlFlv }}$ knock-out (Jnk1 $1^{-\prime-}$; Dong et al., 1998; The Jackson Laboratory), and Mapk $9^{t m l F l v}$ knock-out (Jnk2 ${ }^{-1-}$; Yang et al., 1998; The Jackson Laboratory). For slice culture experiments, CF-1 (Charles River) dams were crossed with hemizygous Dlx5/6-CIE males. Constitutive mutant embryos were generated by crossing $J n k 1^{+/-}$;

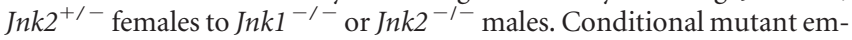
bryos were generated by crossing Jnk1 $1^{f l f l}$; Jnk2 ${ }^{-1-}$ females to $D l \times 5 / 6$ $C I E ; J n k 1^{f l /+} ; J n k 2^{-/-}$males. All animal procedures were performed in accordance to protocols approved by the Institutional Animal Care and Use Committee at West Virginia University.

Fluorescently activated cell sorting and $q R T-P C R$. Methods for fluorescently activated cell sorting (FACS), extracting RNA, synthesizing cDNA, and performing qRT-PCR were reported previously (Meechan et al., 2012). Briefly, cortices from Dlx5/6-CIE + embryos were individually dissociated with papain (Worthington Biochemical), resuspended in FACS buffer, sorted based on endogenous GFP fluorescence, and Dlx5/ 6-CIE $(+)$ and Dlx5/6-CIE $(-)$ sorted samples were homogenized separately in TRIzol reagent (Ambion). Total RNA was isolated and cDNA synthesized using the Improm-II RT kit (Promega). qPCR was conducted using EvaGreen (Bio-Rad) reagent and a CFX384 thermal cycler (Bio-Rad). PCR for $n k k 1$ (F: AGCAGAAGCAAACGTGACAAC/R: GCTGCACACACTATTCCTTGAG) and Jnk2 (F: CCAGTAGGATTGCCTGCTTA/R: TGGTCACATGCATACGAGTC) was performed. Gapdh was used as the endogenous reference gene control. Fold change was determined by the delta-delta CT method (Livak and Schmittgen, 2001).

Organotypic slice cultures. Embryos were collected and dissected in ice-cold complete HBSS (cHBSS; Tucker et al., 2006). Dlx5/6-CIE+ brains were embedded in 3\% low melting point agarose (Fisher Scientific), sectioned at $300 \mu \mathrm{m}$ on a Leica VT1000 S vibratome, transferred to laminin/poly-D-lysine-coated transwell inserts (BD Falcon; Polleux and Ghosh, 2002), and grown at $37^{\circ} \mathrm{C}$ with $5 \% \mathrm{CO}_{2}$ in slice culture media (Tucker et al., 2006) for $24 \mathrm{~h}$. Stock solutions of pharmacological inhibitors [20 mm SP600125 (Enzo Life Sciences), 20 mm AS601245 (Enzo Life Sciences), and $100 \mathrm{~mm}$ U0126 (EMD Chemicals)] were prepared in DMSO, stored at $-20^{\circ} \mathrm{C}$, and added to slice culture media immediately
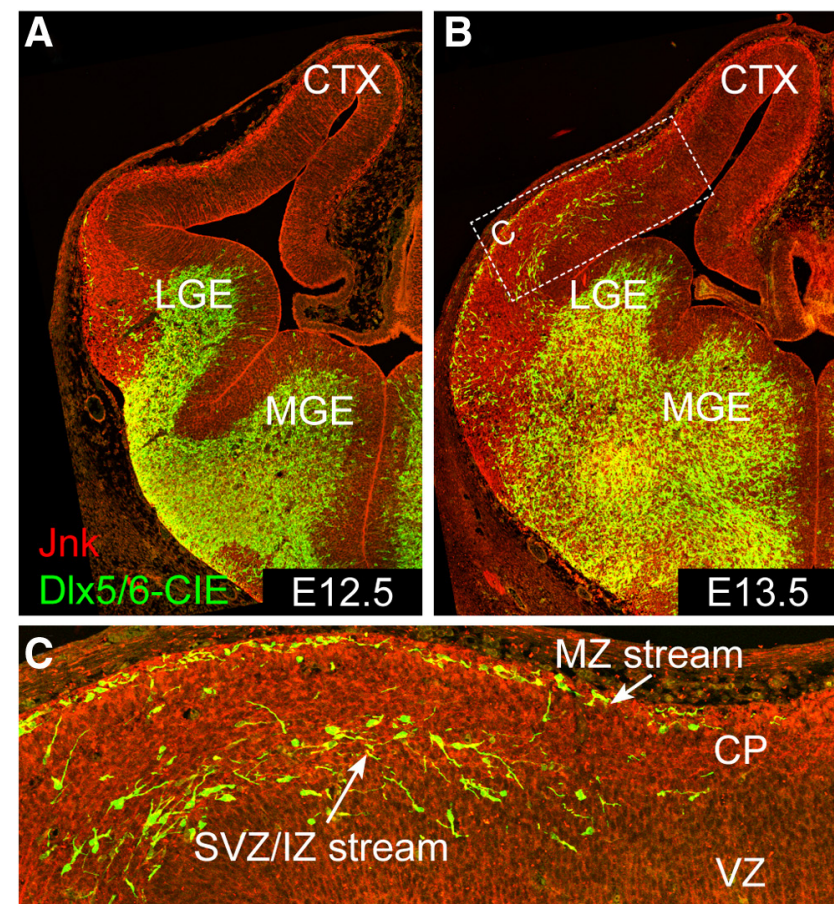

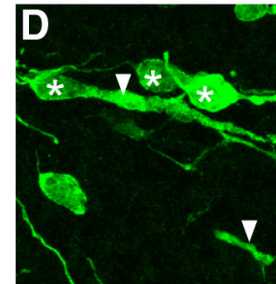

G

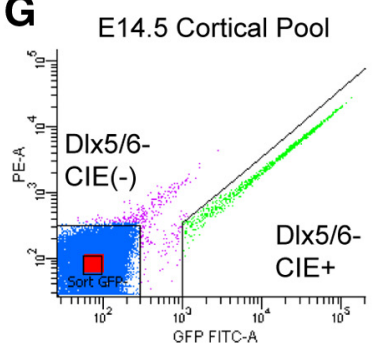

Figure 1. Early arriving cortical interneurons express Jnk proteins. $\boldsymbol{A}-\boldsymbol{F}$, Localization of total Jnk (red) and EGFP (green) expression in D/x5/6-CIE + embryonic brain sections. Jnk proteins are widely expressed in the developing forebrain at E12.5 and E13.5, and are particularly enriched in postmitotic zones. $A$, At E12.5, few Dlx5/6-CIE + cortical interneurons have entered the cortex (CTX) from subcortical locations. B, C, By E13.5, many Dlx5/6-CIE + cortical interneurons have invaded the cortical rudiment and formed streams of migratory cells in the $M Z$ and SVZ/IZ of the nascent cortex. D-F, Dlx5/6-CIE + interneuronal cell bodies (asterisks) and processes (arrowheads) colocalize with total Jnk protein in the E13.5 cortex. G, Separation of DIx5/ 6-CIE $(+)$ and Dlx5/6-CIE $(-)$ cells from a dissociated E14.5 cortex by FACS sorting. Three cortices were independently processed for gene expression analyses by qRT-PCR. $\boldsymbol{H}, \mathrm{qRT}-\mathrm{PCR}$ indicates Jnk1 transcript is enriched twofold in Dlx5/6-CIE $(+)$ cells compared with Dlx5/6$\mathrm{CIE}(-)$ cells (Student's $t$ test; $p=0.02$ ), whereas Jnk2 transcript is uniformly expressed in the two populations. CP, Cortical pool.

before use. DMSO was used as a vehicle control at matching solvent concentrations to inhibitors.

Live imaging experiments. Live vibratome slices were transferred to Millicell cell culture inserts (Millipore) in FluoroDishes (World Precision Instruments) filled with slice culture media containing a 1:1000 dilution of DMSO (for controls) or $20 \mu \mathrm{M}$ SP600125 JNK inhibitor. FluoroDish preparations were immediately transferred to a Zeiss LSM 510 Meta Confocal Microscope with stable environmental controls maintained at $37^{\circ} \mathrm{C}$ with $5 \%$ humidified $\mathrm{CO}_{2}$. Time-lapse $z$-series were 
A

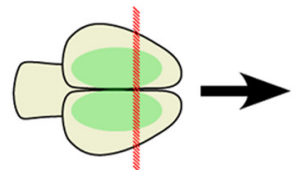

E12.5 Dlx5/6-ClE
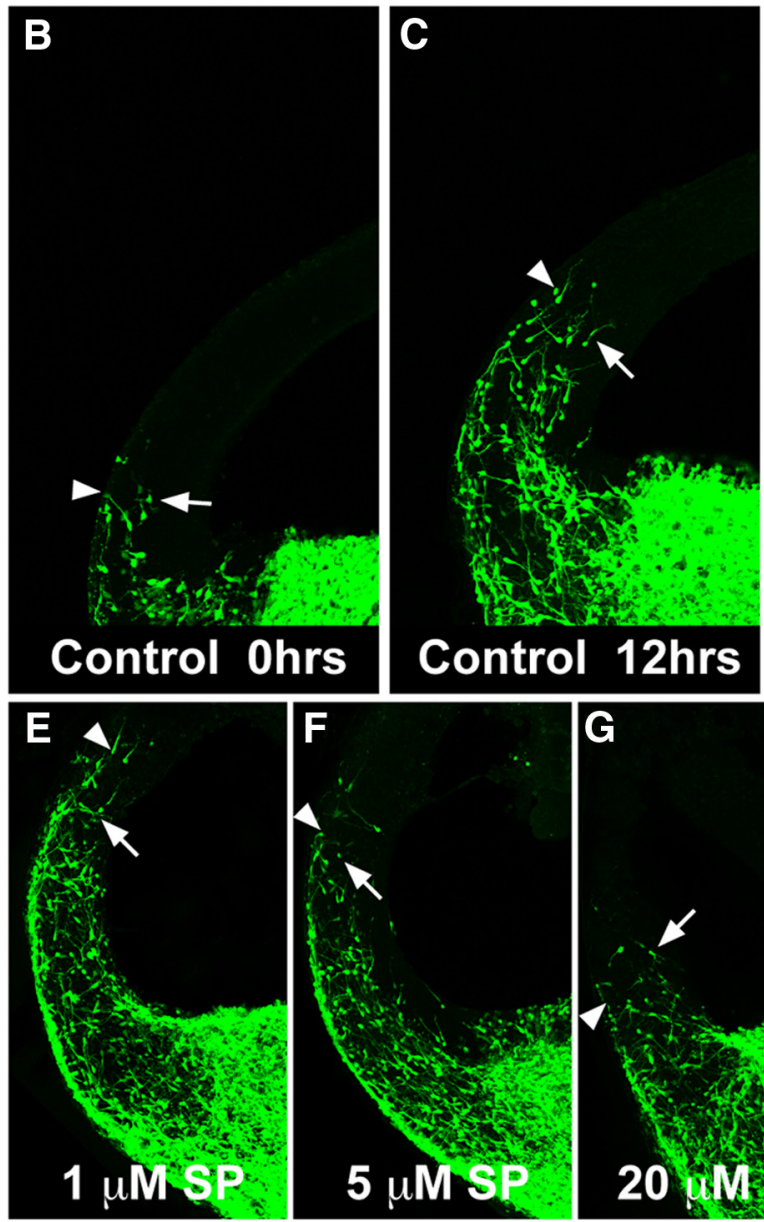

I

Cortical Binning

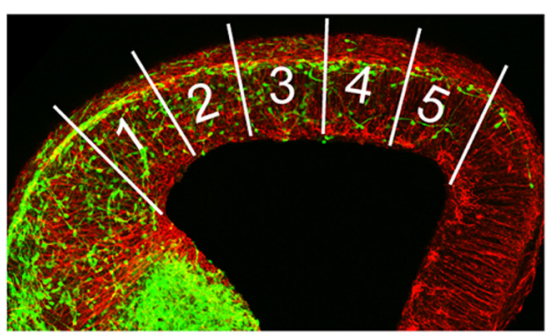

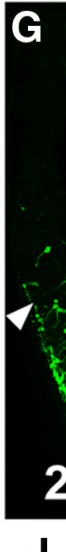

$\mathbf{J}$

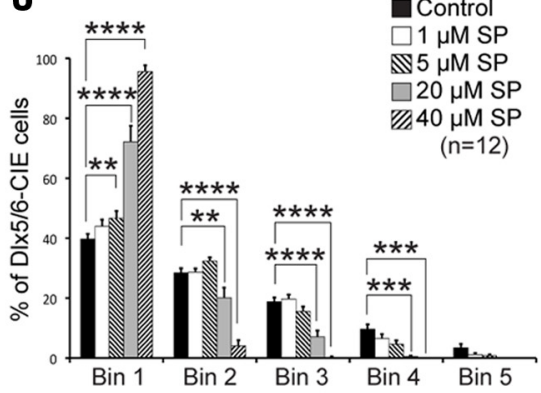

Figure 2. Cortical interneurons require JNK activity to enter the developing cerebral cortex. $\boldsymbol{A}$, Schematic representation of slice culture assay. E12.5 Dlx5/6-CIE + brains are isolated, sectioned, and cultured $24 \mathrm{~h}$ in control or SP600125 (SP) conditions. $\boldsymbol{B}-\boldsymbol{D}$, In control cultures, cortical interneurons robustly migrate into the cortical rudiment during the $24 \mathrm{~h}$ culture period. Migratory front is marked by a flanking arrowhead (MZ region) and arrow (SVZ region) in each image. $\boldsymbol{E}-\boldsymbol{H}$, Entry of cortical interneurons into the cerebral cortex is disrupted by SP600125, a pan-JNK inhibitor, in a dose-dependent fashion. $I$, For quantification of interneuron migration in slice cultures, cortices were segmented into five equidistant bins and the percentages of cortical interneurons appearing in all cortical bins were determined for each section, and averaged across all sections per treatment group. J, Dose-dependent accumulation of cortical interneurons in lateral and decline of interneurons in medial cortical bins following SP600125 treatment. Tw0-way ANOVA reveals statistically significant interactions between treatment and bin location $\left(F_{(16,275)}=57.5 ; p<0.0001\right)$. Differences within bins were determined post hoc by Fisher's LSD tests $\left({ }^{* * *} p<0.0001 ;{ }^{* * *} p \leq 0.0006 ;{ }^{* *} p<0.008\right)$. acquired every $10 \mathrm{~min}$ for $24 \mathrm{~h}$ with an LD Plan-Neofluar $20 \times / 0.4$ Korr objective lens.

MGE explant culture and analysis. Brains from embryonic day 12.5 (E12.5) Dlx5/6-CIE; $J n k 1^{f l /+} ;$ Jnk2 $2^{-/-}$(control) or Dlx5/6-CIE; Jnk1 ${ }^{f l f f l} ;$ Jnk2 ${ }^{-/-}$(conditional double knockout) embryos were removed in ice-cold cHBSS, embedded in 3\% low melting point agarose, and sectioned at $300 \mu \mathrm{m}$. Medial ganglionic eminence (MGE) tissue was isolated from appropriate sections and trimmed to generate subventricular zone-enriched explants. Each brain was processed individually and yielded approximately two MGE explants plus material for genotyping, which was performed retrospectively. A 1:1 collagen-Matrigel mixture was made by preparing a $2 \mathrm{mg} / \mathrm{ml}$ working stock of neutralized collagen (BD Life Sciences; Type 1 Rat Tail High Concentration) in phenol-red free Neurobasal medium (Invitrogen) and diluting it 1:1 with phenol-red free Matrigel (Corning Life Sciences). Ice-cold collagen-Matrigel mixture was added to each well of an eight-well Lab-Tek chambered coverglass, and MGE explants were pipetted into matrix-filled wells. MGE-embedded matrices were allowed to solidify for $45 \mathrm{~min}$ at $37^{\circ} \mathrm{C}$ with $5 \% \mathrm{CO}_{2}$, fed with phenol-red free Neurobasal medium supplemented with GlutaMAX (Invitrogen), penicillin-streptomycin (Thermo Scientific), and B-27 (Invitrogen), and cultured for $2 \mathrm{~d}$. Cultured explants were imaged on a Zeiss LSM 510 confocal microscope at a uniform optical thickness. Area measurements were performed on projected montages in ImageJ. Outgrowth area was determined by subtracting the explant area from the total area occupied by both the explant and migrating cells (Fig. $8 F$ ), and normalized to explant size. Explants were measured blind to genotype and evaluated statistically by a two-tailed unpaired Student's $t$ test using Prism 6 (GraphPad) software.

Immunostaining of slice cultures. Slices were fixed overnight in $4 \%$ paraformaldehyde in $1 \times$ PBS at $4^{\circ} \mathrm{C}$. Slices were rinsed from fixative with $1 \times$ PBS, and blocked overnight at $4^{\circ} \mathrm{C}$ in permeability solution (Polleux and Ghosh, 2002) with 5\% normal goat serum. Rabbit antiGFP (Invitrogen; 1:1500) and mouse antinestin (BD Transduction Laboratories; 1:1000) primary antibodies were diluted in block, applied to slices, and incubated overnight at $4^{\circ} \mathrm{C}$. Slices were thoroughly rinsed in $1 \times \mathrm{PBS}$, and incubated overnight at $4^{\circ} \mathrm{C}$ with secondary antibody solution containing goat anti-rabbit Alexa488 (1:4000; Invitrogen), goat anti-mouse Alexa546 (1:2000; Invitrogen), and the nucleic acid counterstain Draq5 (1:4000; Axxora) diluted in block. Slices were rinsed in $1 \times$ PBS and slide mounted in an aqueous mounting medium containing anti-fade reagent.

Cryosectioning and staining. E13.5 heads were removed and fixed with $4 \%$ paraformaldehyde overnight, rinsed in $1 \times$ PBS, and progressed through a sucrose series $(10,20$, and $30 \%)$. Cryoprotected heads were embedded with TFM Tissue Freezing Medium (Triangle Biomedical) and flash frozen in liquid 
nitrogen-cooled 2-methyl butane. Heads were serially sectioned $(12 \mu \mathrm{m})$ in the coronal plane and slides stored at $-20^{\circ} \mathrm{C}$ before use. Slides were rehydrated with $1 \times$ PBS for $20 \mathrm{~min}$, blocked (as above) for $2 \mathrm{~h}$ at room temperature, and incubated in primary antibody solution (block with primary antibodies) overnight at $4^{\circ} \mathrm{C}$. Primary antibodies included rabbit anti-calbindin (Swant; 1:2000), mouse antinestin (BD; 1:1000), and chicken anti-GFP (Abcam; 1:1500). Slides were rinsed with $1 \times$ PBS, incubated with secondary antibody and nucleic acid counterstain solution (as above) for $2 \mathrm{~h}$ at room temperature, rinsed in $1 \times \mathrm{PBS}$, and coverslipped with an aqueous mounting medium containing anti-fade reagent.

Imaging and analysis. Immunofluorescently labeled slice cultures and cryosections were imaged on a Zeiss LSM 510 Meta Confocal Microscope with a $10 \times$ Fluar or $20 \times$ Plan-Apo objective lens. Confocal micrographs were uniformly adjusted for levels, brightness, and contrast in Adobe Photoshop. For quantification of migration, cortical length was measured from the corticostriatal boundary to the cortical arch of each image, and cortices were segmented into five equidistant bins (Figs. 2, 5). Similarly, for quantification of radial distribution (Fig. 8), a defined region of the lateral cortical wall was cropped from montaged images and equidistantly segmented into six bins from pial to ventricular surfaces. For both studies, the numbers of cells present in each bin were counted and their percentile distributions across all bins were determined for each tissue section. Bin distributions were averaged across sections of the same treatment group or genotype, and statistical significances were determined by two-way ANOVA followed by Fisher's LSD post hoc analyses (GraphPad Prism 6).

$4 \mathrm{D}$ live imaging movies were analyzed using Imaris (Bitplane). Movies (12 control and 12 inhibitor) were evaluated in the first $12 \mathrm{~h}$ and last $12 \mathrm{~h}$ of each recording. Ten interneurons were tracked in each time segment for all movies. Interneurons were selected for tracking if they could be followed for at least $4 \mathrm{~h}$. Tracks were discontinued if a cell remained stationary for 40 contiguous minutes, or if the tracked cell could no longer be unambiguously identified. Average velocity, displacement, and straightness values were obtained for each movie (20 cells). Each treatment group contained 120 cells analyzed from 12 movies $(n=12)$. Twotailed unpaired Student's $t$ tests (GraphPad Prism 6) were used to determine statistical differences between groups.

\section{Results}

Interneurons express Jnk proteins as they first enter the cortical rudiment

We used transgenic mice expressing EGFP under the control of a Dlx5/6 Cre-IRES-EGFP transgene (hereafter referred to as Dlx5/ 6 -CIE) to follow the migration of newly born interneurons into the developing mouse cortex. Dlx5/6-CIE + interneurons first arrive at the entrance to the cortical rudiment on E12.5 (Fig. 1A). By E13.5, Dlx5/6-CIE+ interneurons have crossed the corticos-

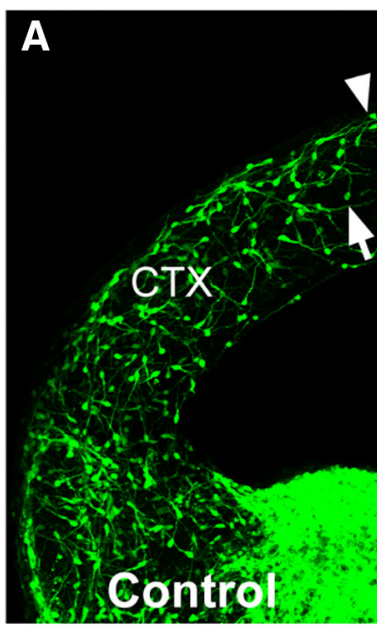

D
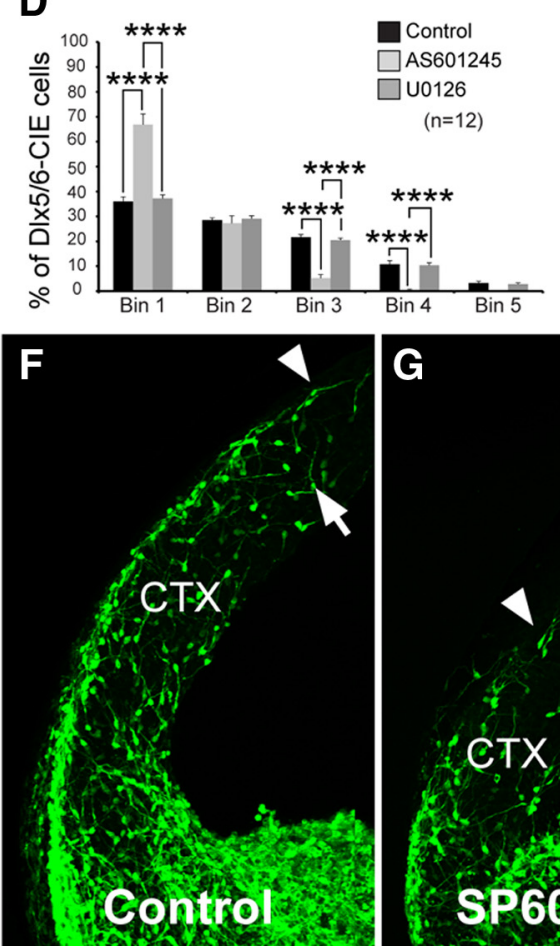

B

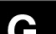

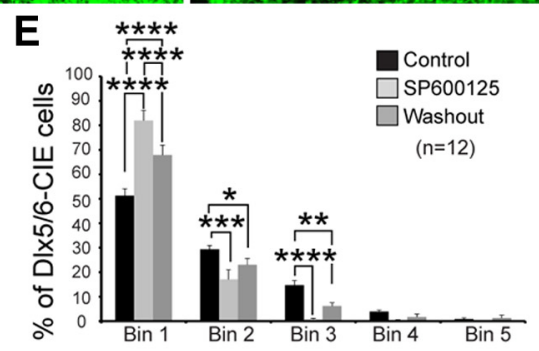

Figure 3. Disruption of cortical interneuron migration by pharmacological inhibition of JNK signaling is specific and reversible. $\boldsymbol{A}-\boldsymbol{D}$, An independent, pan-JNK inhibitor, AS601245 (B), diminishes interneuron entry into the cortex (CTX) compared with control (A) cultures, while blockade of MAPK signaling with the Mek1/2 inhibitor U0126 (C) does not. D, Quantification reveals significant

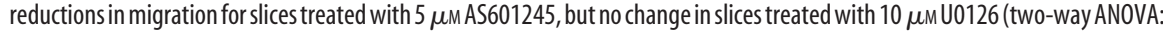
$F_{(8,165)}=15.98 ; p<0.0001$; Fisher'sLSD: $\left.{ }^{* * *} p<0.0001 ;{ }^{* * *} p=0.0002 ;{ }^{* *} p=0.009 ;{ }^{*} p=0.049\right)$. E-H, Cortical interneuron migration recovers after removal of $20 \mu \mathrm{M}$ SP600125. E, Quantification reveals that interneuron migration improves when $20 \mu \mathrm{M}$ SP600125 is washed out and replaced by control medium half way through the $24 \mathrm{~h}$ culture period (two-way ANOVA: $F_{(8,165)}=$ 37.56; $p<0.0001$; Fisher's LSD: $\left.{ }^{* * *} p<0.0001\right)$. $F$, Slice cultured in control medium, rinsed at $12 \mathrm{~h}$, and cultured for an additional $12 \mathrm{~h}$ in control medium. G, Slice cultured in $20 \mu \mathrm{m} \mathrm{SP600125,} \mathrm{rinsed} \mathrm{at} 12 \mathrm{~h}$, and cultured for an additional $12 \mathrm{~h}$ in fresh $20 \mu \mathrm{M}$ SP600125. $\boldsymbol{H}$, Slice cultured in $20 \mu \mathrm{m}$ SP600125, rinsed at $12 \mathrm{~h}$, and cultured for an additional $12 \mathrm{~h}$ in control medium.

triatal boundary and have migrated approximately half the length of the lateral cortical wall (Fig. $1 B, C$ ). As they enter the neocortex, Dlx5/6-CIE + interneurons form two medially oriented migratory streams flanking the emerging cortical plate; an upper marginal zone (MZ) stream and lower subventricular zone/intermediate zone (SVZ/IZ) stream (Fig. 1C). Thus, the first cohort of Dlx5/6-CIE+ cortical interneurons forms streams of migratory cells that rapidly enter the cortical rudiment over a single developmental day.

JNK signaling regulates cell survival (Kuan et al., 1999), radial migration (Westerlund et al., 2011), and axon tract formation 

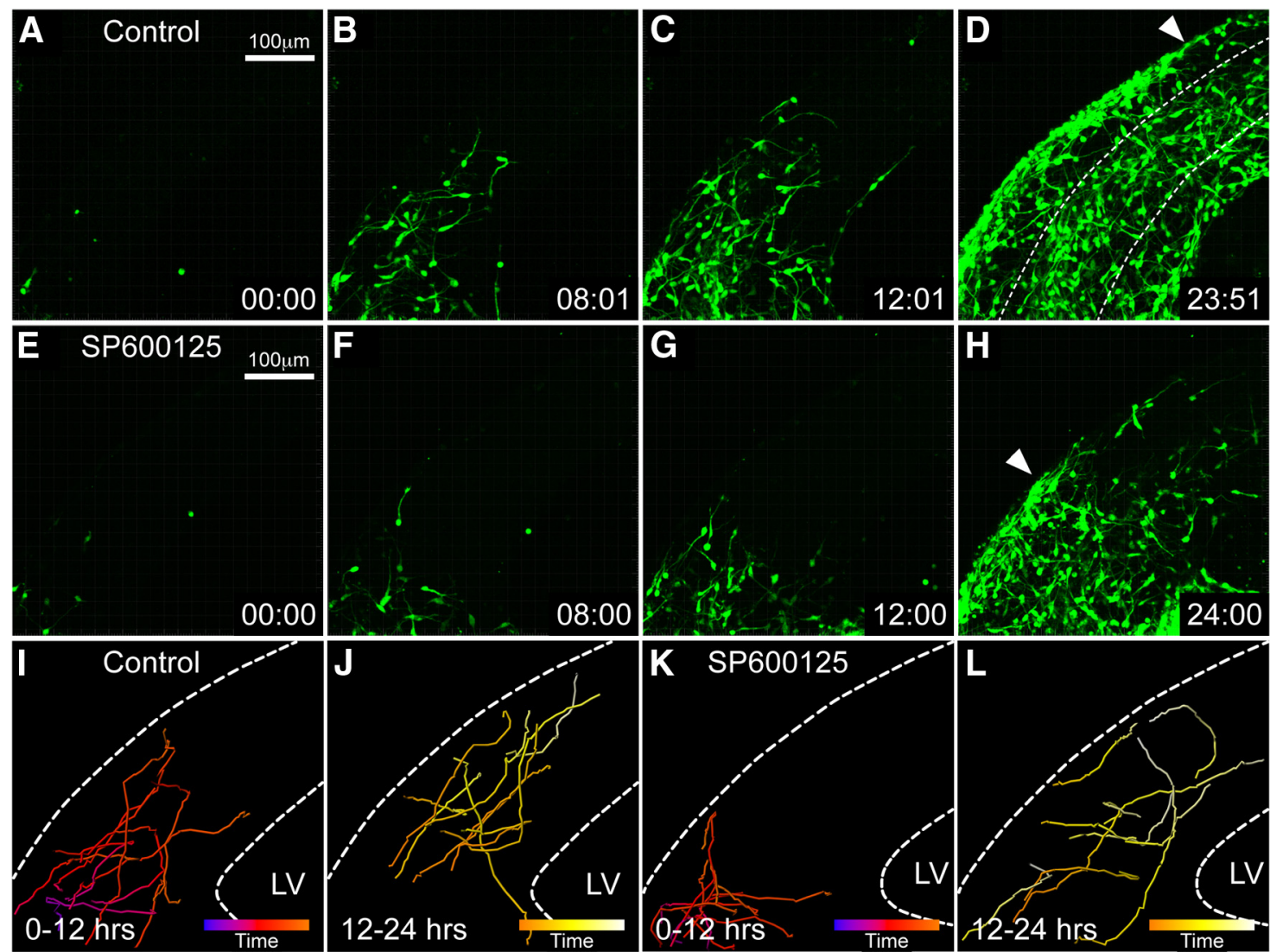

N Track Length (0-12 hrs)

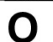

Directionality (0-12 hrs)
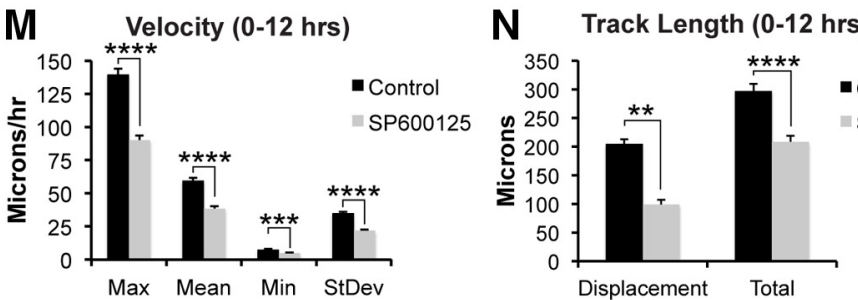

Q Track Length (12-24 hrs)

$\mathbf{P}$

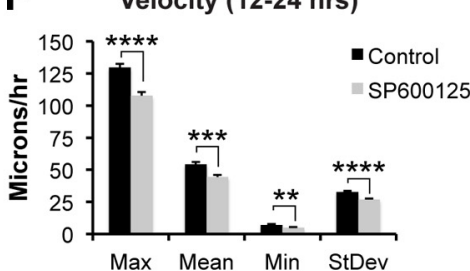

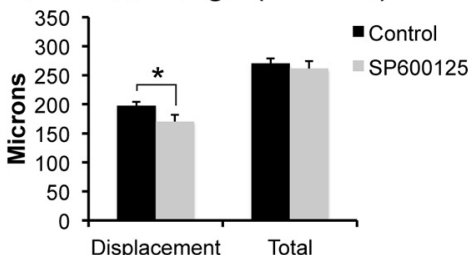

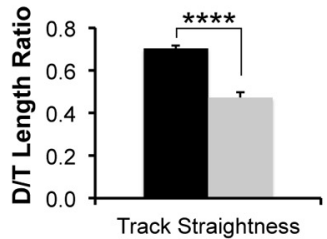

R Directionality (12-24 hrs)

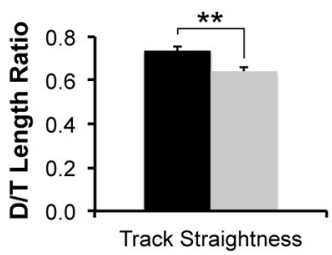

- Control

SP600125

Figure 4. Migratory properties of cortical interneurons are perturbed following SP600125 treatment at E12.5. A-D, In control slices, Dlx5/6-CIE + interneurons robustly migrate through the cortical entry zone over a $24 \mathrm{~h}$ imaging period. Cells travel in MZ region (arrowhead in D) and SVZ region (dashed lines in D) of the section. E-H, In slices treated with $20 \mu \mathrm{m} \mathrm{SP600125,} \mathrm{Dlx5/6-CIE+}$ cells remain motile, but advance slowly and frequently take aberrant trajectories. (ells pile up in MZ (arrowhead in $\boldsymbol{H}$ ), failing to advance further. $\boldsymbol{I}, \boldsymbol{J}$, Tracks from $10 \mathrm{individual}$ cells in the first 12 (I) and last $12(J) h$ of a control movie. Individual cell tracks are long, straight, and medially directed. $\boldsymbol{K}, \boldsymbol{L}$, Tracks from 10 individual cells in the first $12(\boldsymbol{K})$ and last $12(\boldsymbol{L}) \mathrm{h}$ of an inhibitor movie. Cell tracks are short, crooked, and less medially oriented in SP600125-treated slices, particularly in the first $12 \mathrm{~h}$ of imaging. Tracks are pseudocolored by time (bars in $\mathbf{I - L}$ ). $\mathbf{M} \mathbf{- 0}$, Comparison of interneuron migration in SP600125-treated and control slices during the first $12 \mathrm{~h}$ of recordings. SP600125 treatment leads to statistically significant reductions in migratory velocity $(\boldsymbol{M})$, track length $(\boldsymbol{N})$, and track straightness $(\mathbf{O})$ corresponding to directionality. $\boldsymbol{P}-\boldsymbol{R}$, Comparison of interneuron migration in SP600125-treated and control slices in the last $12 \mathrm{~h}$ of recordings. Interneuron migratory velocity $(\boldsymbol{P})$ increases in SP600125-treated slices during the second $12 \mathrm{~h}$ of recording due to waning inhibitor efficacy, but remains significantly reduced from controls. Track length $(\boldsymbol{Q})$ of JNK-inhibited interneurons also increase during the last $12 \mathrm{~h}$, but displacement values remain significantly altered. Directionality $(\boldsymbol{R})$ of interneurons from SP600125-treated slices remains significantly altered in the last $12 \mathrm{~h}$. Significance levels after performing two-tailed unpaired Student's tests are denoted as follows: ${ }^{* * *} p<0.0001,{ }^{* * *} p<0.001$, ${ }^{* *} p<0.01,{ }^{*} p<0.05$.

(Hirai et al., 2006; Yamasaki et al., 2011) in the developing forebrain. However, the extent to which JNK signaling influences the migration of cortical interneurons is unknown. We examined the expression pattern of total Jnk protein between E12.5 and E13.5 to determine whether JNK signaling could regulate the initial migration of cortical interneurons into the cerebral cortex. Jnk protein is widely expressed in the developing forebrain (Fig. $1 A, B)$, but appears to be enriched in the nascent cortical plate and mantle region of the ventral forebrain, where newly generated postmitotic neurons reside. Moreover, Jnk proteins are expressed by tangentially migrating Dlx5/6-CIE + cortical interneurons as they enter the cerebral cortex (Fig. 1D-F). qRT- 


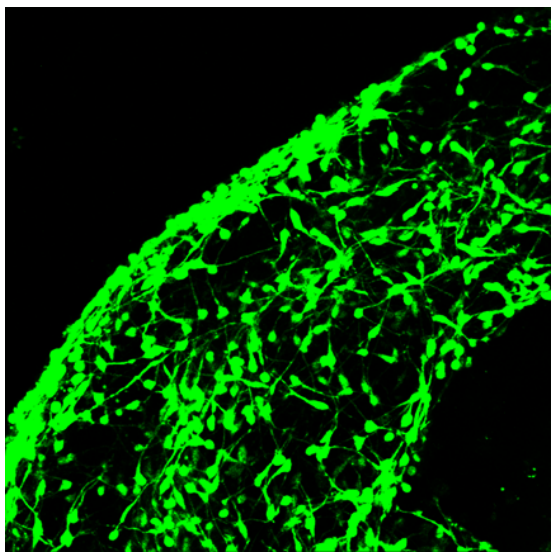

Movie 1. Live imaging of cortical interneuron migration through the cortical entry zone at E12.5 in a control slice (Fig. 4). Video clip 1, Ventrolateral, bottom left; dorsomedial, upper right. Dlx5/6-CIE + cells organize into migratory streams and rapidly invade the nascent cortical rudiment over the $24 \mathrm{~h}$ imaging period. Video clip 2, Cell track 1 (purple spot and tail) represents a Dlx5/6-CIE + cell tracked during the first $12 \mathrm{~h}$ imaging period. Cell 1 maintains a medially directed trajectory, but shifts from SVZ to VZ regions of the slice during the tracking period. Video clip 3, Cell track 2 (red spot and tail) represents a Dlx5/6-CIE + cell tracked during the last $12 \mathrm{~h}$ imaging period. Cell 2 maintains a long, medially directed trajectory, but shifts positions within the SVZ/IZ stream during the tracking period. Spots mark tracked cells, and tails represent where the cell traveled over the last hour of time.

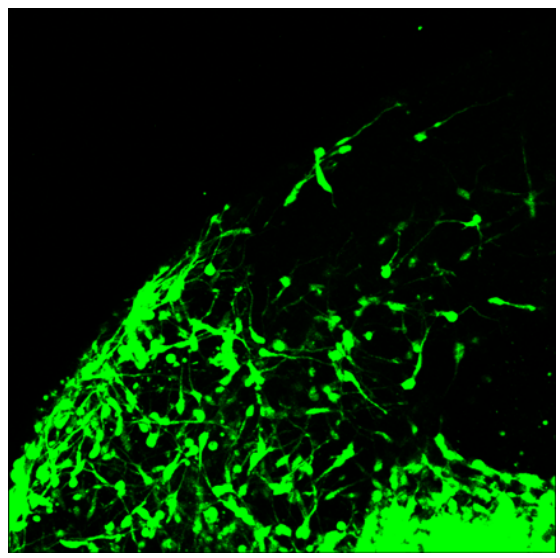

Movie 2. Live imaging of cortical interneuron migration through the cortical entry zone at E12.5 in an SP600125-treated slice (Fig. 4). Video clip 1, Ventrolateral, bottom left; dorsomedial, upper right. DIx5/6-CIE + cells tentatively migrate into the cortical entry zone during the first $12 \mathrm{~h}$ of recording, often migrating slowly into the cortex only to reverse directions and leave. Migration improves during the last $12 \mathrm{~h}$ of recording, but many cells pile up in the MZ stream or spread out in the SVZ/IZ region without advancing past the cortical entry zone. Video clip 2, Cell track 1 (purple spot and tail) represents a Dlx5/6-CIE + cell tracked during the first $12 \mathrm{~h}$ imaging period. Cell 1 migrates a short distance medially, traveling from the SVZ/IZ to the $\mathrm{MZ}$ regions of the slice, only to stall, turn, and migrate back toward the corticostriatal border. Video clip 3, Cell track 2 (red spot and tail) represents a DIx5/6-CIE + cell tracked during the last $12 \mathrm{~h}$ imaging period. Cell 2 migrates in a radial trajectory, traveling from the VZ to the MZ region of the slice, only to turn and migrate laterally, like Cell 1. Spots mark tracked cells, and tails represent where the cell traveled over the last hour of time.

PCR analysis of FACS sorted Dlx5/6-CIE + and Dlx5/6-CIE (-) cells from dissociated E14.5 cortices indicated that $J n k 1$ mRNA is enriched at nearly twofold (average fold change \pm SEM $=1.99 \pm$ 0.22 ) in Dlx5/6-CIE + cortical interneurons, while Jnk2 is uniformly expressed in both populations (Fig. 1H). Thus, Dlx5/6$\mathrm{CIE}+$ cortical interneurons express Jnk proteins as they enter the cortical rudiment and, compared with Dlx5/6-CIE $(-)$ cortical cells, selectively express $J n k 1$ at higher levels.
Interneuron entry into the cortical rudiment requires intact JNK signaling

To evaluate the initial entry of interneurons into the cerebral cortex under controlled conditions, we prepared live-vibratome slices from E12.5 Dlx5/6-CIE + brains and cultured them for $1 \mathrm{~d}$ in vitro (Fig. $2 A$ ). Initially, very few cortical interneurons were present in the cortical rudiment (Fig. 2B). Over the next day in vitro (DIV), Dlx5/6-CIE + cortical interneurons rapidly invaded the lateral aspect of the cerebral cortex (Fig. $2 C, D$ ), recapitulating their cortical entry in vivo (Fig. $1 A-C$ ).

To determine whether interneuron entry into the cerebral cortex depends on intact JNK signaling, we cultured E12.5 Dlx5/ 6 -CIE+ slices for $1 \mathrm{DIV}$ in the presence of different concentrations of SP600125, a selective, reversible, pan inhibitor of JNK signaling (Bennett et al., 2001). At $1 \mu \mathrm{M}$, SP600125 had little effect on interneuron entry into the cortex (Fig. 2E). As the concentration of SP600125 was increased from 5 to $40 \mu \mathrm{M}$, however, a dose-dependent inhibition of interneuron migration into the cerebral cortex was observed (Fig. $2 \mathrm{~F}-\mathrm{H}$ ). To quantify interneuron entry into the cortex, we determined the frequency in which Dlx5/6-CIE + interneurons were found in five equidistant cortical bins (Fig. 2I). When compared with control sections, interneurons from SP600125-treated sections showed a dose-dependent accumulation in the most lateral cortical bin, and dose-dependent reductions in medial bin locations (Fig. $2 J$ ). Thus, pharmacological blockade of JNK signaling prevents the initial entry of Dlx5/6$\mathrm{CIE}+$ cortical interneurons into the cerebral cortex in a dosedependent fashion.

Blockade of interneuron entry into the cerebral cortex by JNK inhibition is specific and reversible

To determine whether disruption of cortical interneuron migration was specific to JNK inhibition, we evaluated whether pharmacological blockade of the MAPK signal transduction cascade had a similar effect on the initial entry of cortical interneurons into the cortical rudiment. Unlike JNK inhibition, U0126, a potent and selective inhibitor of Mek1/2 (Favata et al., 1998), failed to prevent the entry of Dlx5/6-CIE + interneurons into the cerebral cortex (Fig. 3C). Indeed, no differences were found between the advancement of Dlx5/6-CIE + interneurons in slices treated with $10 \mu \mathrm{M}$ U0126 and control slices (Fig. 3D), confirming previous assessments of U0126 on cortical interneuron migration (Polleux et al., 2002).

To further validate the specificity of JNK inhibition on cortical interneuron migration, we cultured E12.5 Dlx5/6-CIE+ slices for $24 \mathrm{~h}$ in the presence of AS601245, an independent pan-inhibitor of JNK signaling (Carboni et al., 2004). Similar to SP600125 treatment, application of AS601245 significantly impaired entry of Dlx5/6-CIE + interneurons into the cortical rudiment (Fig. $3 B, D)$. Indeed, a $5 \mu \mathrm{M}$ concentration of AS601245 impaired interneuron entry to an extent approximating a $20 \mu \mathrm{M}$ concentration of SP600125 (Figs. 2G, 3B). Thus, application of two independent inhibitors of JNK signaling prevented interneuronal migration into the nascent cortical rudiment, while pharmacological inhibition of MAPK signaling had no effect, suggesting JNK signaling, but not MAPK signaling, is required for the initial migration of cortical interneurons into the cortical rudiment.

We next asked whether blockade of interneuron migration into the cerebral cortex by SP600125 treatment was reversible. E12.5 Dlx5/6-CIE + slices were exposed to $20 \mu \mathrm{M}$ SP600125 for $12 \mathrm{~h}$, rinsed, and grown for an additional $12 \mathrm{~h}$ in either control medium ("washout" condition; Fig. $3 \mathrm{H}$ ) or medium containing freshly prepared $20 \mu \mathrm{M}$ SP600125 (“SP600125” condition; Fig. 

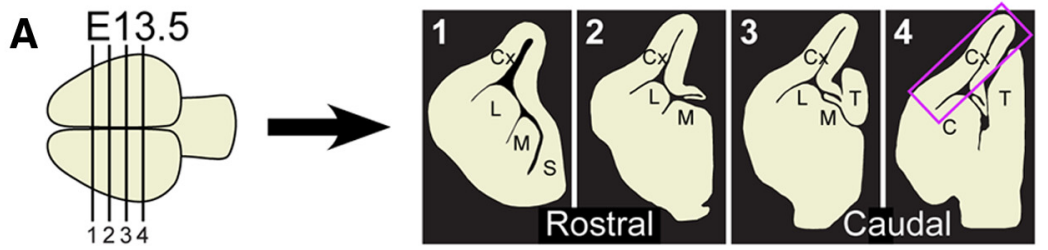

\section{Equidistant Bins}
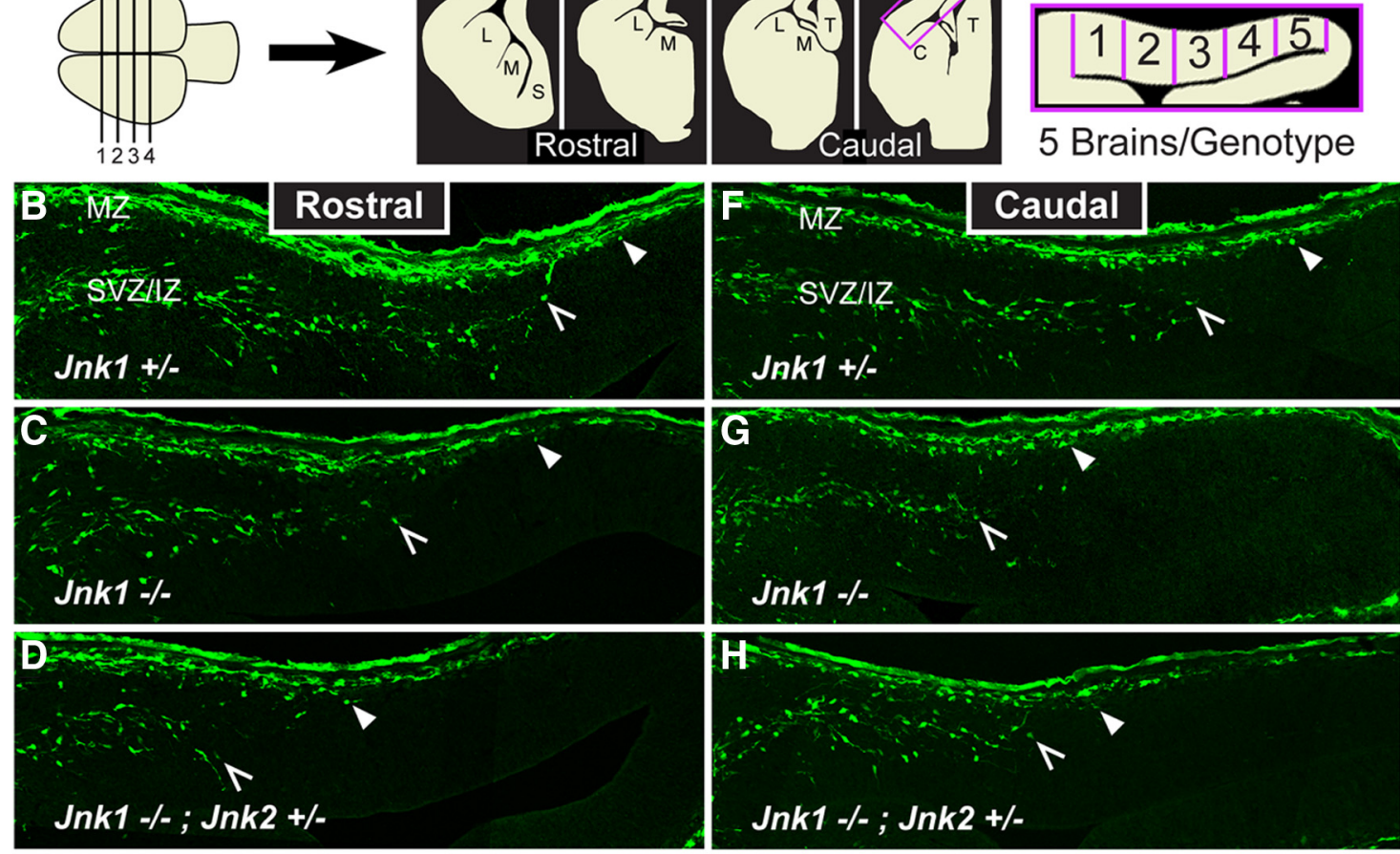

E

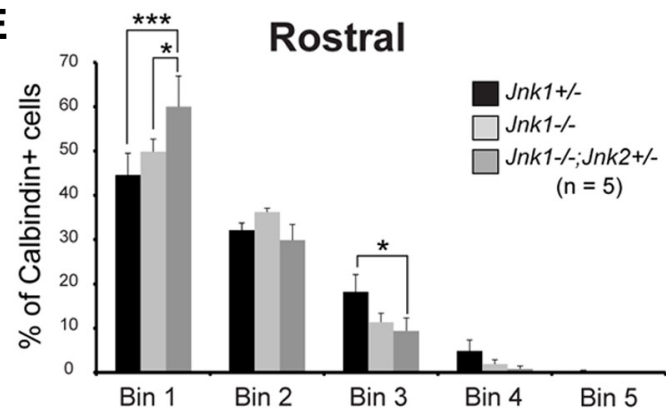

I

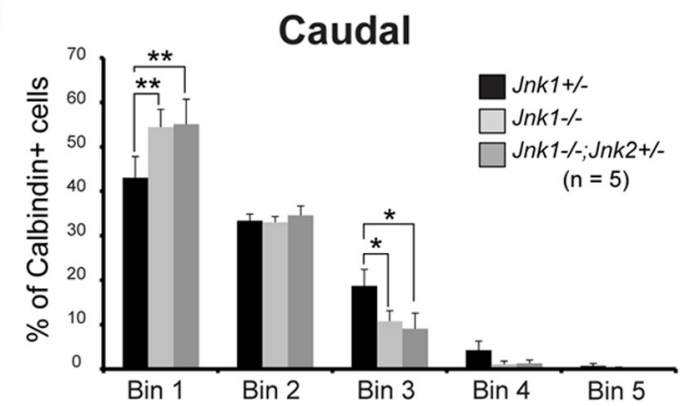

Figure 5. Genetic ablation of Jnk 1 impairs migration of cortical interneurons in vivo. A, Sampling strategy for mutant embryo analyses (Figs. 5-7). Five E13.5 brains were serially sectioned and bilaterally sampled at four rostrocaudal locations per genotype ( $(x$, cortex; L, LGE; M, MGE; C, CGE; S, septum; T, thalamus). Cortices were divided into five equidistant bins, and the percentages of calbindin-positive cells appearing in cortical bins were determined. Data from rostral (slices 1 and 2) and caudal (slices 3 and 4) positions were analyzed separately. $\boldsymbol{B}-\boldsymbol{E}$, Distribution of calbindin-positive cells in $J n k 1^{+/-}(\boldsymbol{B}), J n k 1^{-/-}(\boldsymbol{C})$, and $J n k 1^{-/-} ; \mathrm{Ink} 2^{+/-}(\boldsymbol{D})$ cortices at rostral positions. Furthest extent of interneuron progression is marked by closed (MZ) and open (SVZ/IZ) arrowheads in each image. $\boldsymbol{E}$, Statistically significant interactions were found between genotype and bin location (two-way ANOVA: $\left.F_{(8,60)}=2.742 ; p=0.0120\right)$. Compared with $/ n k 1^{+l-}$ controls, migration of calbindin-positive cortical interneurons was significantly impaired in Jnk $1^{-/-} ;$Jnk2 $2^{+/-}$embryos (Fisher's LSD: ${ }^{* * *} p=0.0005 ;{ }^{*} p=0.019$ in Bin 1,0.042 in Bin 3). F-I, Distribution of calbindin-positive cells in the caudal cortex of $J n k 1^{+/-}(\boldsymbol{F}), J n k 1^{-I-}(G)$, and $J n k 1^{-/-} ; J n k 2^{+/-}$embryonic brains $(\boldsymbol{H}) . \boldsymbol{I}$, Statistically significant interactions were observed between genotype and bin location (two-way ANOVA: $F_{(8,60)}=2.463 ; p=0.0223$ ). Migration of calbindin-positive cortical interneurons was significantly impaired when $J n k 1^{-l-}\left(\right.$ Fisher's LSD: ${ }^{* *} p=0.005 ;{ }^{* *} p<$ 0.049 ) and $J n k 1^{-/-} ;$Ink2 ${ }^{+/-}$cortices (Fisher's LSD: ${ }^{* *} p=0.003 ;{ }^{*} p=0.017$ ) were compared with/nk1 ${ }^{+/-}$controls. LGE, lateral ganglionic eminence.

$3 G$ ). When compared with control slices (Fig. $3 F$ ), slices in both washout and SP600125 conditions displayed impaired migration (Fig. 3G,H). Dlx5/6-CIE+ interneurons showed robust recovery in washout conditions, however, and migrated much further into the cortical rudiment than Dlx5/6-CIE + interneurons in slices continuously exposed to SP600125 (Fig. 3E-H). Thus, SP600125 treatment impairs interneuron entry into the cortex, but this effect is largely reversible upon removal of JNK inhibition.

\section{JNK inhibition impairs directed migration of cortical} interneurons into the cortical rudiment

To further examine consequences of JNK inhibition on the migration of cortical interneurons, time-lapse recordings were made from E12.5 Dlx5/6-CIE+ slices exposed to control medium or medium containing $20 \mu \mathrm{M}$ SP600125. Live images of the cortical entry zone and lateral aspect of the cortical rudiment were acquired every 10 min for $24 \mathrm{~h}$ in control and JNK-inhibited conditions, and migratory properties of Dlx5/6-CIE + cortical interneurons were examined as they entered and navigated the cerebral cortex.

In control slices, Dlx5/6-CIE+ interneurons robustly entered the cortical rudiment and traveled tangentially to fill the lateral cortical wall (Fig. 4A-D; Movie 1, video clip 1). Many Dlx5/6-CIE+ interneurons traversed the cortical entry zone by $12 \mathrm{~h}$ of recording (Fig. 4C), and by $24 \mathrm{~h}, \mathrm{Dlx} 5 / 6$-CIE + interneurons had formed MZ and SVZ/IZ streams on their medial progression through the cortex (Fig. 4D), often migrating beyond the field of view.

In stark contrast, migration of Dlx5/6-CIE + interneurons through the cortical entry zone was severely compromised when slices were treated with $20 \mu \mathrm{M}$ SP600125 (Fig. 4E,H; Movie 2, video clip 1). Dlx5/6-CIE + interneurons remained motile, yet failed to advance in medially oriented trajectories. After $12 \mathrm{~h}$, far fewer Dlx5/6-CIE+ interneurons had entered the cortex (Fig. 
4G) compared with control conditions (Fig. 4C). By 24 h, progression of Dlx5/6$\mathrm{CIE}+$ interneurons into the cortex had improved (Fig. 4H), but failed to match the extent of interneuron migration seen in controls (Fig. 4D), as previously quantified (Fig. 2J). Dlx5/6-CIE + cells often stacked up in the MZ stream (Fig. $4 H$ ), and remained dispersed in the SVZ/IZ region without forming an organized migratory stream.

To quantify changes in migratory behavior, individual Dlx5/6-CIE+ interneurons were analyzed in control and SP600125-treated slices. Representative cell tracks of cortical interneurons in control (Fig. 4I, J; Movie 1, video clips 2-3) and JNK-inhibited (Fig. $4 K, L$ ) conditions are shown for movies depicted in Figure 4. Navigational errors were apparent during the first $12 \mathrm{~h}$ of imaging in SP600125treated slices, when Dlx5/6-CIE + interneurons stall their advancement at the entrance to the cortex, and take short, aberrant paths toward the pial or ventricular surfaces (Fig. 4K; Movie 2, video clip 2). Lengths of individual cell tracks (track length) increased during the last $12 \mathrm{~h}$ of imaging in SP600125-treated slices, but Dlx5/6-CIE + interneurons typically remained misguided (Fig. 4L; Movie 2, video clip 3), and the entire migratory front of cortical interneurons remained less advanced compared with controls.

For statistical comparisons, 240 migratory interneurons from 12 control and 12 SP600125-treated slices were tracked and their trajectories were analyzed. Twenty Dlx5/6-CIE + interneurons were tracked from each slice: 10 during the first $12 \mathrm{~h}$, and 10 during the last $12 \mathrm{~h}$ of imaging. Speed of interneuron advancement significantly declined after SP600125 treatment, with greatest deficits occurring during the first $12 \mathrm{~h}$ period (Fig. $4 M$ ). Maximum, mean, minimum, and SD velocities of Dlx5/6-CIE+ interneurons diminished significantly in SP600125-treated slices (values $=$ avg \pm SEM; Control: $\max =139.6 \pm 4.4$, mean $=$ $59.6 \pm 2.0, \mathrm{~min}=7.6 \pm 0.5, \mathrm{SD}=35.1 \pm 1.0 \mu \mathrm{m} / \mathrm{h} ; \mathrm{SP} 600125$ : $\max =90.3 \pm 3.3$, mean $=38.5 \pm 1.8, \min =4.9 \pm 0.4, \mathrm{SD}=$ $21.8 \pm 0.9 \mu \mathrm{m} / \mathrm{h}$ ). Accordingly, average track lengths from the beginning to ending point of their trajectories (displacement) as well as average cumulative distances traveled (total) were significantly impaired during the first $12 \mathrm{~h}$ period (Control: displacement $=204.6 \pm 8.1$, total $=297.2 \pm 12 \mu \mathrm{m} ;$ SP600125: displacement $=98.9 \pm 8.3$, total $=208.4 \pm 10.7 \mu \mathrm{m}$; Fig. $4 \mathrm{~N})$. Migratory velocities of interneurons in SP600125-treated slices increased during the last $12 \mathrm{~h}$ of imaging (Fig. $4 P$ ), but remained significantly lower than controls (Control: $\max =129.6 \pm 2.9$, mean $=54.3 \pm 1.7, \min =7.0 \pm 0.6, \mathrm{SD}=32.8 \pm 0.8 \mu \mathrm{m} / \mathrm{h}$; SP600125: $\max =107.8 \pm 2.7$, mean $=44.5 \pm 1.6, \min =4.9 \pm$ $0.5, \mathrm{SD}=26.8 \pm 0.8 \mu \mathrm{m} / \mathrm{h}$ ). As velocity increased during the last $12 \mathrm{~h}$ of imaging, so did distances traveled by individual cortical interneurons. Although both displacement and total track lengths increased, displacement length remained reduced to statistically significant levels in the last $12 \mathrm{~h}$ of imaging (Control: displacement $=197.6 \pm 6.4$, total $=270.5 \pm 8.4 \mu \mathrm{m} ;$ SP600125: displacement $=170.6 \pm 11.3$, total $=261.7 \pm 12.6 \mu \mathrm{m}$; Fig. $4 Q$ ).
Directionality of migration as reflected in overall track straightness values, however, was significantly altered in interneurons from SP600125-treated slices during both first (Control: $r=$ $0.70 \pm 0.01 ;$ SP600125: $r=0.47 \pm 0.02)$ and last $12 \mathrm{~h}$ intervals (Control: $r=0.74 \pm 0.02$; SP600125: $r=0.64 \pm 0.02$; Fig. 4O,R).

Improvements observed in migratory speed and track length during the last $12 \mathrm{~h}$ of imaging in SP600125-treated slices likely reflect declining efficacy of the pharmacological inhibitor over the $24 \mathrm{~h}$ imaging period. When interneurons were allowed to grow into the cerebral cortex for $12 \mathrm{~h}$ in control conditions before treatment with SP600125, deficits in interneuron migration were comparable to those observed in acutely treated slices. For example, mean interneuron velocity was reduced to $37.2 \pm 1.2 \mu \mathrm{m} / \mathrm{h}$, which was slightly less than the mean velocity observed during the first $12 \mathrm{~h}$ of SP600125 treatment. Furthermore, to evaluate whether the JNK requirement persisted beyond cortical entry at E12.5, we analyzed migratory properties of control and JNKinhibited cortical interneurons at the leading front of migration in E14.5 cortices. Similar to SP600125 treatment at E12.5, pharmacological inhibition of JNK at E14.5 led to statistically significant reductions in total track length (Control: $267.3 \pm 17.4$; SP600125: $188.1 \pm 8.5 \mu \mathrm{m} ;{ }^{\star} p=0.01$ ), mean velocity (Control: $51.7 \pm 0.8$; SP600125: $\left.32.1 \pm 1.6 \mu \mathrm{m} / \mathrm{h} ;{ }^{* * *} p=0.0004\right)$, and directionality (Control: $0.75 \pm 0.05 ; \mathrm{SP} 600125: 0.56 \pm 0.05 ;{ }^{\star} p=$ $0.04)$. Thus, inhibition of JNK signaling significantly slows the advancement and alters the trajectories of migratory cortical interneurons in the cortical entry zone at E12.5 and at the leading front of migration in E14.5 cortices.

\section{Jnk1 regulates cortical interneuron migration at the cortical entry zone in vivo}

To determine whether loss of Jnk genes compromised migration of cortical interneurons in vivo, we evaluated the distribution of 

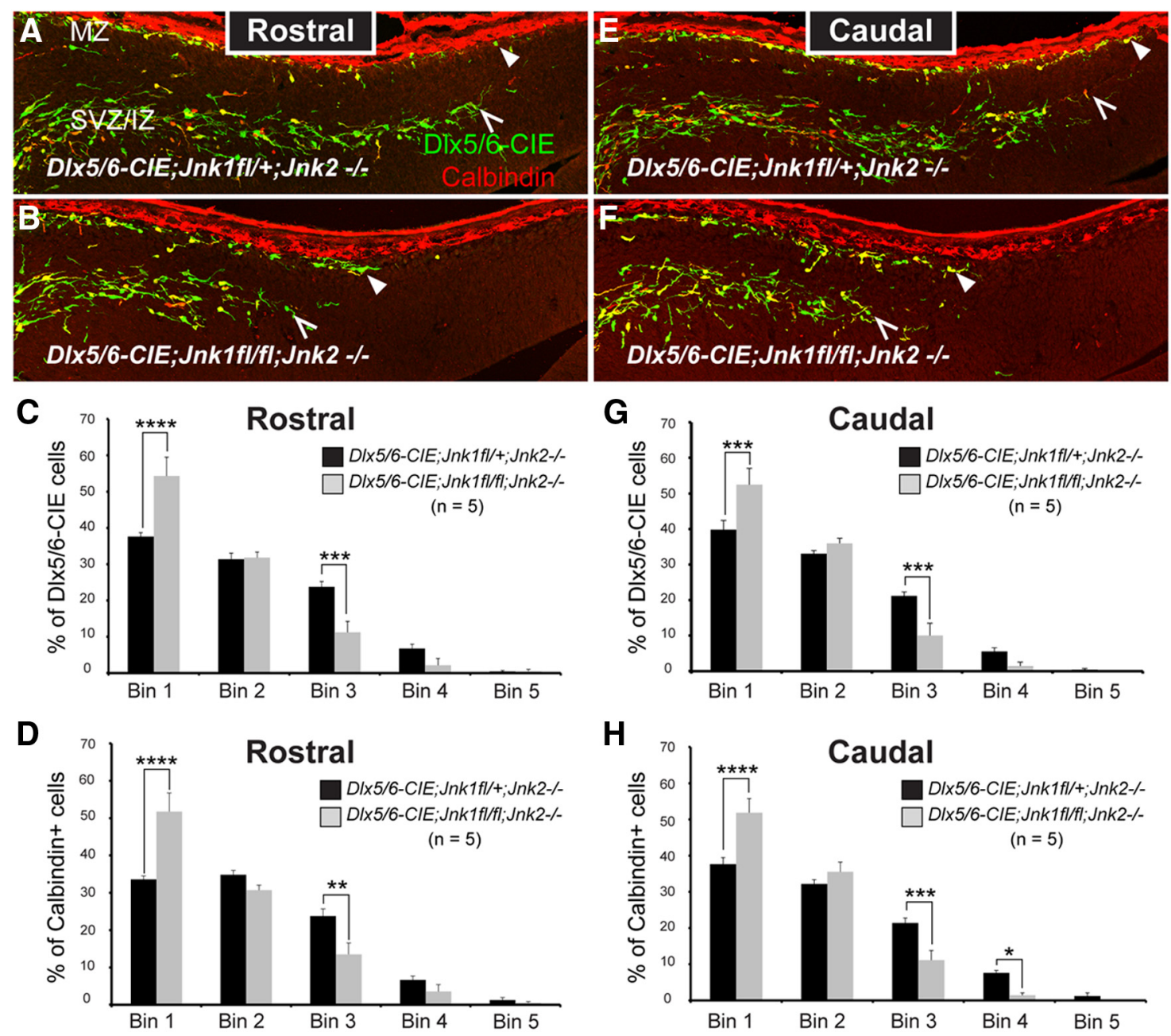

Figure 7. Cortical interneurons have a cell-intrinsic requirement for JNK signaling to migrate through the cortical entry zone in vivo. Jnk1 was conditionally ablated in Dlx5/6-CIE + cells of Jnk2 ${ }^{-I-}$ embryos. A-D, Distribution of Dlx5/6-CIE + (green) and calbindin-positive (red/yellow) cortical interneurons in the rostral cortex of Dlx5/6-CIE; Jnk $7^{f /+} ;$;nk2 ${ }^{-I-}(\boldsymbol{A})$ and $D / x 5 / 6-C I E ;$ $J n k 7^{f l / f l} ;$ Ink ${ }^{-1-}$ embryonic brains $(\boldsymbol{B}) . \boldsymbol{C}, \boldsymbol{D}$, Statistically significant interactions were found between genotype and bin location for Dlx5/6-CIE $+\left(\right.$ two-way ANOVA $F_{(4,40)}=11.55 ; p<0.0001 ; \boldsymbol{C}$ ) and calbindin-positive cortical interneurons (two-way ANOVA $\left.F_{(4,40)}=12.42 ; p<0.0001 ; \boldsymbol{D}\right)$. Migratory advancement of interneurons was significantly impaired in D/x5/6-CIE;/Ink $7^{f / / f t} ;$ Jnk2 ${ }^{-I-}$ cortices (Fisher's LSD: Dlx5/6-CIE: ${ }^{* * *} p<0.0001,{ }^{* * *} p=0.0003$; calbindin: ${ }^{* * * *} p<0.0001,{ }^{* *} p=0.002$ ). $\boldsymbol{E}$-H, Distribution of Dlx5/6-CIE + and calbindin-positive cortical interneurons in the

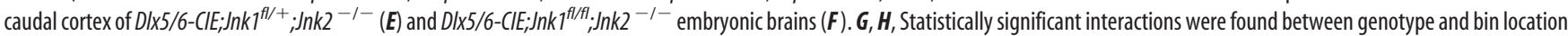
for both Dlx5/6-CIE + (two-way ANOVA: $\left.F_{(4,40)}=8.336 ; p<0.0001 ; \boldsymbol{G}\right)$ and calbindin-positive $(\boldsymbol{H})$ cortical interneurons (two-way ANOVA: $\left.F_{(4,40)}=11.98 ; p<0.0001\right)$. Similar to rostral levels,

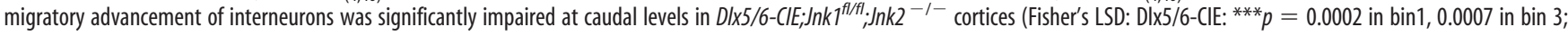
calbindin: ${ }^{* * *} p<0.0001,{ }^{* * *} p=0.0007,{ }^{*} p=0.029$ ).

calbindin-expressing cortical interneurons in an allelic series of Jnk1 and Jnk2 single and combinatorial mutant embryos at E13.5 (Figs. 5, 6), after the initial cohort of interneurons have entered the cerebral cortex. At this age, calbindin expression labels most tangentially migrating interneurons in the cortical rudiment (Anderson et al., 1997). Five embryos of each genotype were coronally sectioned, and the lateral cortical wall was bilaterally sampled at four rostrocaudal locations spanning sections containing the rostral MGE to mid caudal ganglionic eminence (CGE; Fig. 5A). The cortical rudiment was subdivided into five equidistant bins spanning the corticostriatal boundary laterally (Bin1) to cortical arch medially (Bin5; Fig. 5A), and the percentage of calbindin-positive cortical interneurons found in each cortical bin was determined for each section and averaged across all embryos of the same genotype. Since placement of equidistant bins was done with respect to the length of each cortical hemisphere, our sampling strategy accounts for variation in cortical length that might occur between sections.
In $J n k 1^{+/-}$embryos, calbindin-positive interneurons displayed normal tangential migration into the cortical rudiment at both rostral and caudal probe locations (Fig. $5 B, F$ ). However, calbindin-positive interneurons in both MZ and SVZ/IZ streams of $J n k 1^{-1-}$ embryos (Fig. 5C,G) were less advanced than calbindin-positive interneurons in $J n k 1^{+/-}$embryos (Fig. $5 B, F$ ). Accordingly, the proportion of calbindin-positive interneurons increased laterally (Bin1) and declined medially (Bin3-5; Fig. $5 E, I)$. In this and subsequently presented data, statistically significant increases in interneuron abundance are seen in Bin1 and statistically significant decreases in interneuron abundance are seen in Bin3 when migratory deficits are present. Bin2 likely remains unchanged due to transitional normalization occurring between Bins 1 and 3, and while reductions of interneuron abundance occur in Bins $4-5$, they often do not reach statistical significance due to the relatively small percentages of cells found in those bins at E13.5. Although reductions of interneuron migration were apparent at both rostral and caudal probe locations in 
A
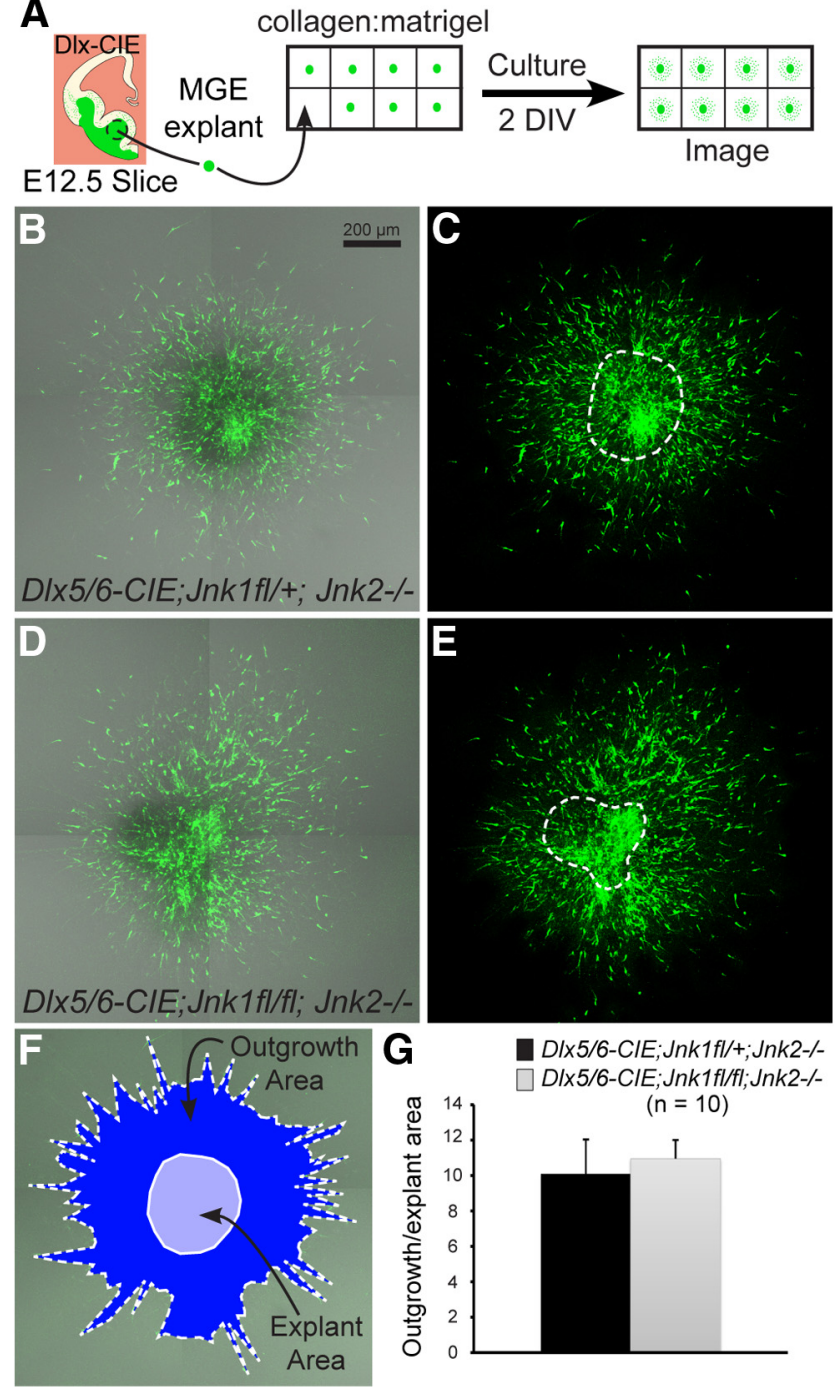

Figure 8. In vitro migration of cortical interneurons from explants of MGE tissue does not rely on JNK signaling. $\boldsymbol{A}$, Illustration of experimental design (see Materials and Methods). $\boldsymbol{B}, \boldsymbol{C}$, Explant of MGE tissue isolated from an E12.5 D/x5/6-CIE;Ink $7^{f l /+} ;$;nk $2^{-1-}$ embryonic brain section and cultured for $2 \mathrm{~d}$ in a collagen:Matrigel matrix. Interneurons have migrated from explant borders in a radial pattern. $\boldsymbol{D}, \boldsymbol{E}, \mathrm{MGE}$ explant from a E12.5 DIX5/6-CIE; Ink $7^{f l / f l}$;Ink2 ${ }^{-1-}$ brain grown under identical conditions, and exhibiting a similar pattern of interneuron outgrowth. $\boldsymbol{F}$, Areas measured from explant shown in $\boldsymbol{B}$ and $\boldsymbol{C}$, illustrating that outgrowth area (dark blue) was determined by subtracting the explant area (light blue) from the total area occupied by both explant and migrating cells. $\mathbf{G}$, Plot representing mean values of normalized outgrowth area (outgrowth area/explant area) for 10 explants from each genotype. DIC images $(\boldsymbol{B}, \boldsymbol{D})$ were used to identify explant boundaries, while perimeters of migrating cells were traced from images containing the EGFP channel alone $(\boldsymbol{C}, \boldsymbol{E})$.

Jnk $1^{-1-}$ embryos, reductions in medial progression only reached statistical significance at caudal probe locations (Fig. 5E,I).

To determine whether reduction of $J n k 2$ in combination with loss of $J n k 1$ exacerbated migratory phenotypes of cortical interneurons, we analyzed the distribution of calbindin-positive cortical interneurons in embryos lacking Jnk1 and a single genomic copy of $J n k 2\left(J n k 1^{-/-} ; J n k 2^{+/-}\right)$, since $J n k 1 / 2$ double nulls die before interneuron migration into the cerebral cortex (Kuan et al., 1999). In Jnk1 $1^{-/-}$; Jnk2 $2^{+/-}$embryos, advancement of calbindin-positive interneurons in both $\mathrm{MZ}$ and SVZ/IZ streams was significantly diminished relative to $J n k 1^{+/-}$controls at both rostral and caudal probe locations (Fig. $5 D, H$ ). Statistically significant interactions were found between genotype and bin position at rostral and caudal probe locations (Fig. 5E,I), suggesting loss of $J n k 1$ alone and loss of $J n k 1$ combined with reduction of $J n k 2$ inhibits tangential progression of cortical interneurons in vivo. At caudal locations, calbindin-positive interneurons in $J n k 1^{-/-} ; J n k 2^{+/-}$and $J n k 1^{-/-}$embryos were comparably regressed (Fig. $5 G-I$ ), but at rostral locations, calbindin-positive interneurons in $\mathrm{Jnk1}^{-/-}$; $n \mathrm{k} 2^{+/-}$embryos were significantly less advanced than those in $J n k 1^{-1-}$ embryos (Fig. 5C-E). To ensure that potential differences in brain size did not influence interpretation of our results, we measured absolute cortical lengths from every section we analyzed and found no statistically significant differences in cortical length between genotypes by one-way ANOVA $\left(J n k 1^{+/-}=937.8 \pm 17.4, J_{n k} 1^{-1-}\right.$ $\left.=1005.0 \pm 21.0, J n k 1^{-/-} ; \mathrm{Jnk} 2^{+/-}=935.4 \pm 27.6 \mu \mathrm{m}\right)$. Thus, at rostral positions, reducing Jnk2 in combination with the loss of $J n k 1$ has a greater impact on cortical interneuron migration than loss of $J n k 1$ alone.

To determine whether Jnk2 plays a comparable role to $J n k 1$ in the initial migration of cortical interneurons in vivo, we generated embryos with the reciprocal $J n k 2^{+/-}, J n k 2^{-l-}$, and $J n k 2^{-l-}$; $J n k 1^{+/-}$genotypes, and evaluated tangential progression of calbindin-positive cortical interneurons at E13.5. Normal migration of cortical interneurons was observed in Jnk $2^{+/-}$embryos at both rostral (Fig. $6 A, D$ ) and caudal (Fig. $6 E, H$ ) probe locations, and their cortical bin distribution closely matched $J n k 1^{+/-}$embryos (Fig. $5 E, I$ ). Unlike in $J n k 1^{-1-}$ embryos, however, tangential progression of calbindin-positive interneurons was completely unperturbed in $J n k 2^{-1-}$ embryos at rostral (Fig. $6 B, D)$ and caudal probe locations (Fig. $6 F, H$ ). Moreover, removing a genomic copy of $J n k 1$ in the context of $J n k 2$ deletion did not alter the advancement of calbindin-positive cortical interneurons (Fig. 6C,D, G,H). Neither MZ nor SVZ streams were regressed in $J n k 2^{-l-}$ or $J n k 2^{-l-} ; J n k 1+1-$ embryos relative to migratory streams in $J n k 2^{+/-}$controls (Fig. $6 A-G$ ), and the distribution of calbindin-positive cortical interneurons in cortical bins were nearly identical across all three genotypes at rostral and caudal locations (Fig. 6D,H). As before, cortical rudiment lengths were consistent between genotypes $\left(\right.$ Jnk2 ${ }^{+/-}=1032.6 \pm$ 22.0, Jnk2 $2^{-l-}=1033.2 \pm 27.6, J_{n k 2} 2^{-l-} ; \mathrm{Jnk} 1^{+/-}=1014.5 \pm 9.3$ $\mu \mathrm{m})$. Apparently, retaining a single genomic copy of $J n k 1$ is sufficient to leave cortical interneuron migration intact and unperturbed in the Jnk2 $2^{-1-}$ background at E13.5.

\section{Cortical interneurons have a cell-intrinsic requirement for Jnk1 in vivo}

Genetic ablation of $J n k 1$ disrupts the initial migration of interneurons into the cortical rudiment, but since $J n k 1$ is expressed in both migratory cortical interneurons and noninterneuronal cells of the developing cortex (Fig. 1), the requirement for JNK signaling in regulating cortical interneuron migration may not be cell autonomous. Indeed, radial migration of cortical projection neurons is accelerated in $J n k 1^{-1-}$ embryos (Westerlund et al., 2011), which could lead to nonautonomous disruptions in the tangential migration of cortical interneurons. To determine whether the initial cohort of cortical interneurons has a cellintrinsic requirement for JNK signaling to migrate into the cortical rudiment, we used mice expressing the Dlx5/6-CIE transgene to conditionally ablate Jnk1 within cortical interneurons in vivo. Since migration of cortical interneurons was completely unperturbed after constitutive loss of $J n k 2^{-1-}$ (Fig. 6), but reduction of $J n k 2$ in $J n k 1$ nulls exacerbated interneuron migratory phenotypes (Fig. 5), we conditionally ablated Jnkl in interneurons of $J n k 2^{-1-}$ embryos. This allowed evaluation of 

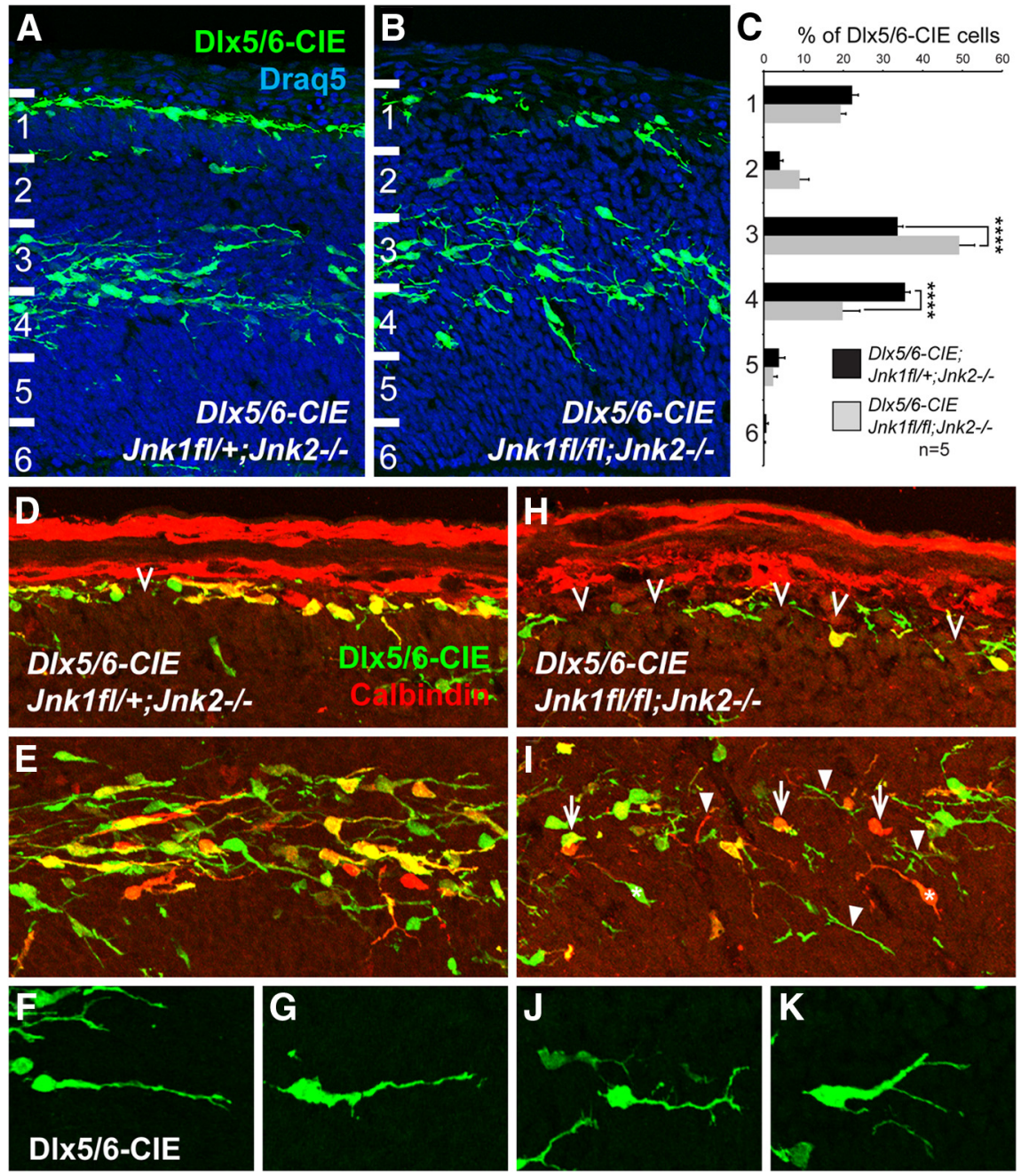

Figure 9. Cortical interneurons lacking Jnk1 and Jnk2 disperse from migratory streams and adopt branched morphologies in vivo. $\boldsymbol{A}-\boldsymbol{C}$, Radial distribution of Dlx5/6-CIE + cells (green) in the lateral cortex of E13.5 D/x5/6-CIE; Jnk $7^{f / /+} ; J n k 2^{-/-}(\boldsymbol{A})$ and $D / \times 5 / 6-\left(I E ; / n k 7^{f l / f l} ; J n k 2^{-1-}\right.$ embryos (B). Equivalently sized cortical probes were equidistantly binned along the radial axis (Bin $1=\mathrm{MZ}-$ Bin $6=\mathrm{VZ}$ ) and the percentage of Dlx5/6-CIE + cells appearing in each bin was determined ( $n=5$ embryos/ genotype). C, Radial distribution of Dlx5/6-CIE + cells is altered in D/x5/6-CIE;/nk $7^{f / f f l}$;Jnk2 ${ }^{-1-}$ cortices (two-way ANOVA: $F_{(5,48)}$ $=13.26 ; p<0.0001)$. Proportionately fewer Dlx5/6-CIE + cells are located in bin 4 (SVZ region; Fisher's $L S D={ }^{* * * *} p<0.0001$ ), while more are located in bin 3 (IZ region; Fisher's LSD $\left.={ }^{* * * *} p<0.0001\right)$. D-G, Dlx5/6-CIE + (green) and calbindin-positive cells (red/yellow) from E13.5 D/x5/6-CIE;Jnk $7^{f / /} ;$;nk ${ }^{-1-}$ cortices. D, Cortical interneurons within the MZ travel in a coherent stream with few gaps (open arrowhead). $\boldsymbol{E}$, Interneurons within the cortical SVZ/IZ stream bare medially directed (image right) leading processes. $\boldsymbol{F}-\boldsymbol{G}$, Examples of isolated Dlx5/6-CIE + cells with simple, unbranched leading processes. $\boldsymbol{H}-\boldsymbol{K}$, Dlx5/6-CIE + (green) and calbindin-positive cells (red/yellow) from E13.5 Dlx5/6-(IE;Ink $7^{f / / f l} ;$ Ink ${ }^{-1-}$ cortices. $\boldsymbol{H}$, Mutant MZ streams are patchy, containing many gaps (open arrowheads). I, Cortical interneurons are loosely organized within the mutant SVZ. Many interneuronal cell bodies (arrows) have no apparent leading processes, and many interneuronal processes (arrowheads) have no apparent cell bodies, suggesting many interneurons are orthogonally positioned to the plane of section. Interneurons with attached processes (asterisks) are often nonmedially directed. $J, K$, Dlx $5 / 6$-CIE + cells from mutant cortices often have branched morphologies. Also note aberrant branching of interneurons in $\boldsymbol{B}$ and $\boldsymbol{I}$ compared with $\boldsymbol{A}$ and $\boldsymbol{E}$.

interneurons that were completely deficient in both $J n k 1$ and Jnk2, which would otherwise be impossible due to the midgestation embryonic lethality of constitutive Jnk1/2 double mutation.

We predicted Dlx5/6-CIE;Jnk1 $1^{f l /+} ;$ Jnk2 $2^{-1-}$ embryos would not have deficiencies in interneuron migration, since interneuron migration was unperturbed in $J n k 2^{-1-}$; Jnk $1+1-$ embryos. Indeed, normal tangential migration of cortical interneurons was observed at E13.5 in Dlx5/6-CIE;Jnk1 ${ }^{f l /+} ; J n k 2^{-/-}$embryos (Fig. $7 A, E)$. We analyzed Dlx5/6-CIE + cortical interneurons, as well as the calbindin-expressing cohort of cortical interneurons, which constitute most of the Dlx5/6$\mathrm{CIE}+$ cells in the cortical rudiment at E13.5. Both populations of labeled cells appeared normally distributed within the cortex of Dlx5/6-CIE; Ink1 $1^{f l /+} ;$ Jnk2 $2^{-1-}$ embryos at rostral (Fig. 7C,D) and caudal probe locations (Fig. $7 G, H$ ). In $D l x 5 / 6$ CIE;Ink1 $1^{f l f l}$;Jnk2 $2^{-l-}$ embryos, however, both Dlx5/6-CIE+ and calbindinpositive cortical interneurons were significantly regressed in their cortical advancement at rostral (Fig. $7 B-D$ ) and caudal (Fig. $7 F-H$ ) probe locations compared with interneurons in control brains. The distribution of interneurons within Dlx5/6-CIE; Jnk1 $1^{f l f l} ; J n k 2^{-1-}$ cortices was significantly altered from control $D l \times 5 / 6$ CIE;Ink $1^{f l++} ;$ Jnk2 $2^{-1-}$ cortices at rostral (Fig. $7 C, D$ ) and caudal probe locations (Fig. $7 G, H)$. Similar to $J n k 1^{-/-}$and Jnk $1^{-/-}$; Ink $2^{+/-}$embryos, cortical interneurons accumulated laterally and diminished medially within Dlx5/6-CIE; $J n k 1^{f l f l} ; J n k 2^{-/-}$cortices, with the largest statistical differences between genotypes occurring in Bin1 and Bin3 (Fig. $7 C, D, G, H)$. Distributions of cortical interneurons within Dlx5/6-CIE; Jnk1 $1^{f l f l}$; $J n k 2^{-1-}$ embryos were comparable to Jnk1 $1^{-/-}$; Jnk2 $2^{+/-}$embryos, since both rostral and caudal portions of their trajectories were significantly regressed relative to their respective controls (Fig. 5E,I). This finding is consistent with Jnk2 loss of function enhancing a $J n k 1^{-1-}$ migratory phenotype at rostral probe locations. Cortical lengths were not significantly different between control and conditional Jnk1/2 double knock-out embryos (Dlx5/ 6-CIE;Ink1 ${ }^{f l /+} ; J n k 2^{-l-}=949.1 \pm 50.1$, Dlx5/6-CIE; Jnk1 $1^{f l f l} ;$ Jnk2 $2^{-l-}=922.7 \pm$ $56.0 \mu \mathrm{m})$. Collectively, these data suggest the initial cohort of cortical interneurons has a cell-intrinsic requirement for JNK signaling-largely mediated through the activity of $J n k 1$, but partially compensated for by $J n k 2$ when $J n k 1$ is lost-in migrating through the cortical entry zone in vivo.

Although our results indicate cortical interneurons have a cell-intrinsic requirement for JNK signaling to appropriately enter the cerebral cortex, there are two possible explanations for this requirement: (1) JNK signaling could intrinsically enhance the migratory capacity of cortical interneurons or (2) JNK signaling could be required by cortical interneurons to sense or respond to environmental cues located in the cortical rudiment. To distinguish between these possibilities, we cultured explants of MGE tissue from E12.5 control or conditional double knock-out embryos in a reduced, serum-free in vitro environment devoid of cortical cues (Fig. $8 A$ ). MGE explants isolated from either Dlx5/6-CIE; Ink fll+ $_{\text {flnk2 }}^{-l-}$ (Fig. $8 B, C)$ or Dlx5/6-CIE;Jnk1 $1^{f l f l} ; J n k 2^{-1-}$ (Fig. 8D,E) forebrain slices produced Dlx5/6-CIE + migratory interneurons that dis- 
persed in a radial pattern from the explant margins. When migratory outgrowth was measured blind to genotype (Fig. $8 F$ ), no statistical differences in interneuron migration were observed between $D l \times 5 / 6$ CIE; Ink1 $1^{f l /+} ; J n k 2^{-l-}$ and Dlx5/6-CIE; $J n k 1^{f l f l} ; J n k 2^{-1-}$ explants of MGE tissue (Fig. 8G). These data strongly suggest that JNK signaling does not act in cortical interneurons to autonomously promote or enhance their motility.

\section{Integrity of cortical migratory streams depends on JNK signaling in vivo}

Since we observed statistically significant deficits in the entry of cortical interneurons into the cortical rudiment after conditionally ablating Jnk1 from interneurons of Jnk2-null mutants in vivo (Fig. 7), but failed to see migratory anomalies in the absence of cortical cues in vitro (Fig. 8), we hypothesized that cortical interneurons have a cell-intrinsic requirement for JNK signaling to navigate the early cortical rudiment in vivo. To determine whether JNK-deficient cortical interneurons are misrouted when first entering the cerebral cortex, we quantified the radial distribution of cortical interneurons within the lateral cortical wall of $D l \times 5 / 6$ CIE;Jnk1 $1^{f l++}$;nk2 $2^{-/-}$control and Dlx5/6-CIE;Jnk1 $1^{f l f l} ; J n k 2^{-/-}$ mutant embryos at E13.5 (Fig. 9A-C). Six equidistant bins were made along the radial axis of the lateral cortex (adjacent to the corticostriatal boundary), and the percentage of Dlx5/6-CIE+ cortical interneurons appearing in Bins 1 (MZ) through 6 (ventricular Zone; VZ) were determined for each genotype. In $D l \times 5$ / 6-CIE;Jnk1 ${ }^{f l+} ;$ Jnk2 $2^{-/-}$embryos, Dlx5/6-CIE+ interneurons were predominantly distributed in the MZ (Bin 1) and SVZ/IZ (Bins 3-4) streams (Fig. 9A,C). In Dlx5/6-CIE; Jnk1 $1^{f l f l}$;Jnk2 ${ }^{-1-}$ embryos, however, the proportions of Dlx5/6-CIE + interneurons located in the MZ stream (Bin 1) and SVZ portion of the SVZ/IZ stream (Bin 4) were diminished compared with control cortices (Fig. $9 B, C$ ). In addition, the percentage of Dlx5/6-CIE+ interneurons in the IZ (Bins 2-3) was elevated in conditional double mutants compared with controls (Fig. 9 B, C). When compared statistically, distributions of Dlx5/6-CIE + migratory interneurons within Bins 1-6 were significantly altered between Dlx5/6-CIE; Ink1 $1^{f l /+} ;$ Jnk2 $2^{-l-}$ and Dlx5/6-CIE;Ink1 $1^{f l f l} ;$ Jnk2 $2^{-1-}$ genotypes with the largest shifts occurring in Bins 3 and 4 (Fig. $9 C$ ), suggesting JNK-deficient interneurons were dispersing from the SVZ.

Moreover, repositioning of migratory interneurons within the radial axis of the Dlx5/6-CIE; Jnk1 ${ }^{f l f l} ;$ Jnk $2^{-/-}$mutant cortex was accompanied by diminished integrity to both $\mathrm{MZ}$ and SVZ/IZ migratory streams. The MZ and SVZ/IZ streams of Dlx5/ 6-CIE; Ink $1^{f l+} ;$ Jnk2 $2^{-1-}$ control embryos (Fig. 9D,E) were consistently more cohesive than MZ and SVZ/IZ streams of Dlx5/ 6-CIE;Ink1 ${ }^{\text {flfl }}$; Ink2 $2^{-1-}$ mutant embryos (Fig. 9H,I). Dlx5/6$\mathrm{CIE}+$ and calbindin-positive cortical interneurons were more loosely organized in Dlx5/6-CIE; Jnk1 ${ }^{\text {flflf }}$; $n k 2^{-1-}$ cortices, leading to frequent gaps within MZ and SVZ/IZ streams (Fig. $9 H, I$ ). Leading processes of cortical interneurons within the SVZ/IZ stream of Dlx5/6-CIE; Jnk $1^{f l /+} ; J n k 2^{-/-}$cortices were typically straight and medially oriented (Fig. 9E), while disordered and
Caudal Cortex at E13.5
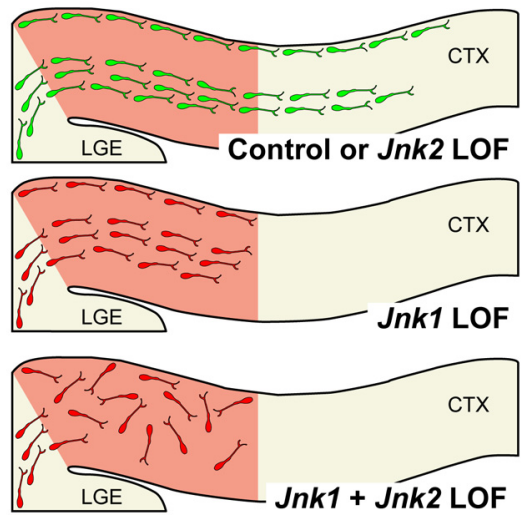

\section{CTX}

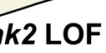

CTX

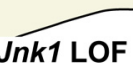

CTX

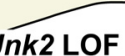

LGE

Figure 10. Migration of cortical interneurons through the cortical entry zone in vivo requires intact JNK signaling, largely mediated by interneuron expressed Jnk1. Schematic drawings of the cortical rudiment at E13.5; ventrolateral is left, dorsomedial CIE. $n k k^{f l /+} \cdot \ln k 2^{-1-}$ resents JNK-dependent cortical "entry zone". LOF, loss of function. Control Jnk1 $1^{+/}$, Ink2 (middle right), but not rostral locations (middle left). pletely devoid of both $J n k 1$ and $J n k 2$ are abnormal positioned in the radial axis of the cortex, disrupting the integrity of $M Z$ and SVZ/IZ streams, and exhibit branching anomalies.

nontangentially oriented in the SVZ/IZ stream of Dlx5/6-CIE; $J n k 1^{f l f f l} ;$;nk2 $2^{-l-}$ mutant cortices (Fig. 9I). In addition, interneuronal processes were often noncontiguous with their cell bodies (Fig. 9I), suggesting migratory orientations of Dlx5/6-CIE; $n k k 1^{f l}$ $f l ; n k 2^{-l-}$ interneurons were frequently orthogonal to the coronal plane of section. Finally, processes of cortical interneurons in Dlx5/6-CIE;Jnk1 $1^{f l f l} ; J n k 2^{-l-}$ embryos were often more highly branched (Fig. 9J,K) than those of control interneurons (Fig. $9 F, G)$. Thus, conditional ablation of $J n k l$ from interneurons of $J n k 2^{-I-}$ embryos disrupts the radial distribution of cortical interneurons at the entrance to the cortical rudiment, diminishes the integrity of MZ and SVZ/IZ migratory streams, and leads to morphological alterations in migratory cortical interneurons. Collectively, these data suggest cortical interneurons have an intrinsic requirement for JNK signaling in navigating the cortical entry zone in vivo.

\section{Discussion}

In the current study, we identified novel and essential roles for JNK signaling in cortical interneuron migration. We established that cortical interneurons express Jnks as they enter the cerebral cortex and, compared with noninterneuronal cortical cells, have enriched expression of Jnkl. Using ex vivo slice cultures, we demonstrated pharmacological blockade of JNK results in dosedependent disruption of interneuron migration into the cerebral cortex, and that JNK-inhibited cortical interneurons slow their advancement and take aberrant trajectories as they navigate the cortical entry zone at E12.5. JNK inhibition at E14.5 similarly perturbed migratory properties of cortical interneurons, indicating their requirement for JNK persists at later embryonic stages. In vivo analyses of single and multi-allelic mutations of two Jnk genes revealed that loss of $J n k 1$ impairs cortical interneuron migration, while loss of Jnk2 does not, unless Jnk2 is reduced in Jnk1 nulls. Finally, we showed conditional deletion of $J n k 1$ in interneurons of $J n k 2$ nulls recapitulates migratory deficits observed in constitutive $J n k 1^{-/-} ; J n k 2^{+/-}$embryos, and in addition, disrupts migratory streams and cortical interneuron morphology. 
Despite this clear intrinsic requirement for JNK activity in vivo, JNK-deficient interneurons migrate normally in vitro, suggesting JNK regulates cortical interneuron migration in response to cortical guidance cues. Together, our results suggest that cortical interneurons have an intrinsic requirement for $J n k 1$ to enter and navigate the developing cerebral cortex, making the JNK signaling pathway a key intracellular mediator of cortical interneuron migration in vivo.

\section{JNKs and neuronal migration}

JNK signaling orchestrates diverse cellular processes in the developing nervous system including cell survival (Kuan et al., 1999; Sabapathy et al., 1999), axon elongation and stability (Hirai et al., 2006; Wang et al., 2007; Yamasaki et al., 2011), axon guidance (Qu et al., 2013), and radial migration (Hirai et al., 2006; Wang et al., 2007; Westerlund et al., 2011; Yamasaki et al., 2011). The extent to which individual Jnk genes have distinct functions remains to be elucidated (Haeusgen et al., 2009; Yamasaki et al., 2012). Here, we report Jnk1 plays a greater role than Jnk2 in cortical interneuron migration. This may either reflect functional differences between $J n k 1$ and $J n k 2$, or unequal expression of $J n k 1$ and $J n k 2$ in migratory cortical interneurons. The contribution of $J n k 3$ to cortical interneuron migration is currently unknown.

Radial migration in the developing cortex relies on JNK signaling, yet it is unclear whether $J n k 1, J n k 2$, and $J n k 3$ play equivalent roles. Deletion of upstream JNK activators including Map3k12 (Hirai et al., 2006), Map2k4 (Wang et al., 2007), or Map2k7 (Yamasaki et al., 2011) inhibits radial migration. Surprisingly, deletion of $J n k 1$ has the opposite effect: radial migration into the cortical plate is accelerated in Jnk1 nulls (Westerlund et al., 2011). These conflicting results could be explained if $J n k 2$ and/or $J n k 3$ play opposite roles to $J n k 1$ in radial migration, since deletion of upstream JNK activators would diminish activity of all available Jnk isoforms, whereas single deletion of $J n k 1$ would not. We find pharmacological inhibition of all JNK activity, as well as genetic deletion of $J n k 1$ or $J n k 1$ and $J n k 2$ together, inhibits tangential progression of cortical interneurons. These results indicate that at least Jnk1 and Jnk2 act in parallel to promote cortical interneuron migration in vivo. Thus, both radial and tangential migration requires Jnk1-mediated signaling, but $J n k 1$ function exerts opposite effects on radially versus tangentially migrating neuroblasts. Differential expression of downstream JNK targets may underlie the molecular bases of these distinctions. For example, the microtubule regulatory protein SCG10/stathmin-2 is a major downstream effector of Jnk1 in radially migrating neuroblasts (Westerlund et al., 2011); cortical interneurons may rely on other JNK substrates, however.

\section{JNK signaling at the "cortical entry zone"}

Our data suggest the first cohort of cortical interneurons requires JNK activity to enter and navigate the lateral cortical rudiment. We call this segment of their migratory pathway the cortical entry zone, which bridges the subcortical telencephalon and neocortex. Extracellular guidance cues located at the cortical entry zone likely direct and concentrate cortical interneurons into newly emerging migratory streams. Indeed, $\mathrm{Cxcl} 12$ is highly expressed within the SVZ/IZ at the cortical entry zone (Stumm et al., 2003; 2007; Tiveron et al., 2006; López-Bendito et al., 2008), and is required for interneuron migration into the cortex via the SVZ/IZ stream (Tiveron et al., 2006; López-Bendito et al., 2008). Thus, cortical interneurons respond to extracellular guidance cues located at the cortical entry zone to correctly navigate the nascent cerebral cortex, and our data suggest JNK signaling crit- ically regulates the spatial and temporal precision for which this occurs.

We provide substantial genetic evidence indicating that JNK signaling regulates interneuron migration at the cortical entry zone in vivo (Fig. 10). Constitutive ablation of $J n k 1$ diminishes cortical interneuron migration at E13.5, with significant reductions in migration occurring at caudal cortical locations. Loss of $J n k 2$ alone or loss of $J n k 2$ with heterozygous reduction of $J n k 1$ has no discernable effect, however, suggesting a single genomic copy of $J n k 1$ is sufficient to maintain normal migratory properties of cortical interneurons-even in the absence of Jnk2. In contrast, heterozygous reduction of $J n k 2$ in $J n k 1$ nulls impairs cortical interneuron migration more than loss of $J n k 1$ alone, since tangential migration into both rostral and caudal portions of their cortical trajectory are compromised. Thus, compared with Jnk1, Jnk2 plays a relatively minor role in cortical interneuron migration, and its influence is only apparent rostrally when $J n k 1$ function is lost. Conditional deletion of $J n k 1$ in interneurons of $J n k 2$ nulls, which completely eliminates $J n k 1$ and $J n k 2$ function in cortical interneurons, significantly impairs cortical interneuron migration throughout the rostrocaudal axis of the developing cortex. Not only is cortical entry compromised when Jnkl and $J n k 2$ function is eliminated from cortical interneurons, but MZ and SVZ/IZ streams are less cohesive, radial positioning of migratory interneurons is altered, and migratory cortical interneurons are more highly branched. In a reduced in vitro environment, however, JNK-deficient interneurons migrate normally, suggesting cortical interneurons require JNK to sense or respond to cortical guidance cues rather than promote motility. Together, our in vivo genetic, ex vivo pharmacologic, and in vitro migration data suggest cortical interneurons have a cellintrinsic requirement for JNK signaling to guide their migration into and within the developing cerebral cortex.

Two cell biological processes control tangential migration of cortical interneurons: (1) dynamic remodeling of the leading process through branching and elongation and (2) translocation of the nucleus into the leading process, or nucleokinesis. Both events require precise molecular control of actin and microtubule cytoskeleton (Bellion et al., 2005; Godin et al., 2012), and must be coordinated in a stepwise fashion to orient migration toward chemoattractant guidance cues (Martini et al., 2009). Although cellular and molecular mechanisms underlying the requirement for JNK in migrating cortical interneurons are currently unknown, we hypothesize JNK signaling acts as a molecular bridge between cortically encountered guidance cues and cytoskeletal machinery driving migration. One potential mediator is doublecortin, a microtubule-associated protein regulated by JNK phosphorylation (Gdalyahu et al., 2004; Jin et al., 2010), and required for proper branching, nucleokinesis, and directed migration of cortical interneurons (Kappeler et al., 2006; Friocourt et al., 2007). The degree to which extracellular guidance cues activate JNK signaling to modulate doublecortin, or other regulators of cytoskeletal dynamics in migrating cortical interneurons, such as Lis1 (Gopal et al., 2010), p27 ${ }^{\text {Kip1 }}$ (Godin et al., 2012), or Rac-GTPases (Tahirovic et al., 2010; Tivodar et al., 2014), remains to be determined.

\section{Relevance to neuropsychiatric disorders}

Cortical interneurons are cellular targets for the pathogenesis of several developmental disorders of cortical connectivity, including schizophrenia and autism ( Marín, 2012). Recent work in a mouse model of 22q11.2 deletion syndrome (22q11.2DS), which confers significant genetic risk for schizophrenia and autism in 
humans (Murphy et al., 1999; Fine et al., 2005; Niklasson et al., 2009), has revealed that embryonic migratory deficits in cortical interneurons lead to laminar repositioning of parvalbuminpositive interneurons in adult cortices (Meechan et al., 2009, 2012). Similar to JNK-deficient interneurons, cortical interneurons in 22q11.2DS brains display delayed cortical entry at E13.5 and irregularities in their migratory streams (Meechan et al., 2009,2012 ). Interneuron migratory deficiencies in 22q11.2DS mice are cell autonomous and largely result from reductions in the chemokine receptor Cxcr4 (Meechan et al., 2012). Independent analysis of a similar mouse model confirmed that functional defects in Cxcl12/Cxcr4 signaling underlie interneuron deficiencies in 22q11.2DS mice (Toritsuka et al., 2013), and implicated $D g c r 8$ as the mediator. It is currently unknown whether genes within the 22q11.2 locus, including $D g c r 8$, interact with the JNK pathway, but similarities in interneuron migratory deficits are highly suggestive of a connection. One plausible explanation is that Cxcl12-Cxcr4 signaling activates JNK to guide interneuron migration and promote migratory stream integrity at the cortical entry zone, but this hypothesis remains to be tested.

Uncovering cellular and molecular mechanisms underlying the pathogenesis of cortical connectivity disorders is essential for developing effective strategies for their treatment. Our current work identifies the JNK signaling pathway, and Jnkl in particular, as a major regulator of interneuron migration in vivo-making the JNK pathway a novel candidate to consider in the etiology of cortical circuit disorders and a target for potential therapeutic interventions.

\section{References}

Anderson SA, Eisenstat DD, Shi L, Rubenstein JL (1997) Interneuron migration from basal forebrain to neocortex: dependence on Dlx genes. Science 278:474-476. CrossRef Medline

Bellion A, Baudoin JP, Alvarez C, Bornens M, Métin C (2005) Nucleokinesis in tangentially migrating neurons comprises two alternating phases: forward migration of the Golgi/centrosome associated with centrosome splitting and myosin contraction at the rear. J Neurosci 25:5691-5699. CrossRef Medline

Bennett BL, Sasaki DT, Murray BW, O'Leary EC, Sakata ST, Xu W, Leisten JC, Motiwala A, Pierce S, Satoh Y, Bhagwat SS, Manning AM, Anderson DW (2001) SP600125, an anthrapyrazolone inhibitor of Jun N-terminal kinase. Proc Natl Acad Sci U S A 98:13681-13686. CrossRef Medline

Carboni S, Hiver A, Szyndralewiez C, Gaillard P, Gotteland JP, Vitte PA (2004) AS601245 (1,3-benzothiazol-2-yl(2-\{ 2-(3-pyridinyl)ethyl amino\}-4 pyrimidinyl) acetonitrile): a c-Jun NH2-terminal protein kinase inhibitor with neuroprotective properties. J Pharmacol Exp Ther 310:25-32. CrossRef Medline

Das M, Jiang F, Sluss HK, Zhang C, Shokat KM, Flavell RA, Davis RJ (2007) Suppression of p53-dependent senescence by the JNK signal transduction pathway. Proc Natl Acad Sci U S A 104:15759-15764. CrossRef Medline

Dong C, Yang DD, Wysk M, Whitmarsh AJ, Davis RJ, Flavell RA (1998) Defective T cell differentiation in the absence of Jnk1. Science 282:20922095. CrossRef Medline

Faux C, Rakic S, Andrews W, Britto JM (2012) Neurons on the move: migration and lamination of cortical interneurons. Neurosignals 20:168189. CrossRef Medline

Favata MF, Horiuchi KY, Manos EJ, Daulerio AJ, Stradley DA, Feeser WS, Van Dyk DE, Pitts WJ, Earl RA, Hobbs F, Copeland RA, Magolda RL, Scherle PA, Trzaskos JM (1998) Identification of a novel inhibitor of mitogen-activated protein kinase kinase. J Biol Chem 273:18623-18632. CrossRef Medline

Fine SE, Weissman A, Gerdes M, Pinto-Martin J, Zackai EH, McDonaldMcGinn DM, Emanuel BS (2005) Autism spectrum disorders and symptoms in children with molecularly confirmed 22q11.2 deletion syndrome. J Autism Dev Disord 35:461-470. CrossRef Medline

Flames N, Long JE, Garratt AN, Fischer TM, Gassmann M, Birchmeier C, Lai C, Rubenstein JL, Marín O (2004) Short- and long-range attraction of cortical GABAergic interneurons by neuregulin-1. Neuron 44:251-261. CrossRef Medline
Friocourt G, Liu JS, Antypa M, Rakic S, Walsh CA, Parnavelas JG (2007) Both doublecortin and doublecortin-like kinase play a role in cortical interneuron migration. J Neurosci 27:3875-3883. CrossRef Medline

Gdalyahu A, Ghosh I, Levy T, Sapir T, Sapoznik S, Fishler Y, Azoulai D, Reiner O (2004) DCX, a new mediator of the JNK pathway. EMBO J 23:823832. CrossRef Medline

Godin JD, Thomas N, Laguesse S, Malinouskaya L, Close P, Malaise O, Purnelle A, Raineteau O, Campbell K, Fero M, Moonen G, Malgrange B, Chariot A, Metin C, Besson A, Nguyen L (2012) p27(KiP1) is a microtubule-associated protein that promotes microtubule polymerization during neuron migration. Dev Cell 23:729-744. CrossRef Medline

Gopal PP, Simonet JC, Shapiro W, Golden JA (2010) Leading process branch instability in Lis $1(+/-)$ nonradially migrating interneurons. Cereb Cortex 20:1497-1505. CrossRef Medline

Haeusgen W, Boehm R, Zhao Y, Herdegen T, Waetzig V (2009) Specific activities of individual c-jun n-terminal kinases in the brain. Neuroscience 161:951-959. CrossRef Medline

Hirai S, Cui de F, Miyata T, Ogawa M, Kiyonari H, Suda Y, Aizawa S, Banba Y, Ohno S (2006) The c-Jun N-terminal kinase activator dual leucine zipper kinase regulates axon growth and neuronal migration in the developing cerebral cortex. J Neurosci 26:11992-12002. CrossRef Medline

Jin J, Suzuki H, Hirai S, Mikoshiba K, Ohshima T (2010) JNK phosphorylates Ser332 of doublecortin and regulates its function in neurite extension and neuronal migration. Dev Neurobiol 70:929-942. CrossRef Medline

Kappeler C, Saillour Y, Baudoin JP, Tuy FP, Alvarez C, Houbron C, Gaspar P, Hamard G, Chelly J, Métin C, Francis F (2006) Branching and nucleokinesis defects in migrating interneurons derived from doublecortin knockout mice. Hum Mol Genet 15:1387-1400. CrossRef Medline

Kuan CY, Yang DD, Samanta Roy DR, Davis RJ, Rakic P, Flavell RA (1999) The Jnk1 and Jnk2 protein kinases are required for regional specific apoptosis during early brain development. Neuron 22:667-676. CrossRef Medline

Kunde SA, Rademacher N, Tzschach A, Wiedersberg E, Ullmann R, Kalscheuer VM, Shoichet SA (2013) Characterisation of de novo MAPK10/JNK3 truncation mutations associated with cognitive disorders in two unrelated patients. Hum Genet 132:461-471. CrossRef Medline

Lavdas AA, Grigoriou M, Pachnis V, Parnavelas JG (1999) The medial ganglionic eminence gives rise to a population of early neurons in the developing cerebral cortex. J Neurosci 19:7881-7888. Medline

Li G, Adesnik H, Li J, Long J, Nicoll RA, Rubenstein JL, Pleasure SJ (2008) Regional distribution of cortical interneurons and development of inhibitory tone are regulated by Cxcl12/Cxcr4 signaling. J Neurosci 28:10851098. CrossRef Medline

Livak KJ, Schmittgen TD (2001) Analysis of relative gene expression data using real-time quantitative PCR and the 2(T)(-Delta Delta C) method. Methods 25:402-408. CrossRef Medline

López-Bendito G, Sánchez-Alcañiz JA, Pla R, Borrell V, Picó E, Valdeolmillos M, Marín O (2008) Chemokine signaling controls intracortical migration and final distribution of GABAergic interneurons. J Neurosci 28 : 1613-1624. CrossRef Medline

Marín O (2012) Interneuron dysfunction in psychiatric disorders. Nat Rev Neurosci 13:107-120. CrossRef Medline

Marín O (2013) Cellular and molecular mechanisms controlling the migration of neocortical interneurons. Eur J Neurosci 38:2019-2029. CrossRef Medline

Marín O, Yaron A, Bagri A, Tessier-Lavigne M, Rubenstein JL (2001) Sorting of striatal and cortical interneurons regulated by semaphorinneuropilin interactions. Science 293:872-875. CrossRef Medline

Martini FJ, Valiente M, López Bendito G, Szabó G, Moya F, Valdeolmillos M, Marín O (2009) Biased selection of leading process branches mediates chemotaxis during tangential neuronal migration. Development 136:4150. CrossRef Medline

Meechan DW, Tucker ES, Maynard TM, LaMantia AS (2009) Diminished dosage of 22q11 genes disrupts neurogenesis and cortical development in a mouse model of 22q11 deletion/DiGeorge syndrome. Proc Natl Acad Sci U S A 106:16434-16445. CrossRef Medline

Meechan DW, Tucker ES, Maynard TM, LaMantia AS (2012) Cxcr4 regulation of interneuron migration is disrupted in 22q11.2 deletion syndrome. Proc Natl Acad Sci U S A 109:18601-18606. CrossRef Medline

Miyoshi G, Hjerling-Leffler J, Karayannis T, Sousa VH, Butt SJ, Battiste J, Johnson JE, Machold RP, Fishell G (2010) Genetic fate mapping reveals 
that the caudal ganglionic eminence produces a large and diverse population of superficial cortical interneurons. J Neurosci 30:1582-1594. CrossRef Medline

Murphy KC, Jones LA, Owen MJ (1999) High rates of schizophrenia in adults with velo-cardio-facial syndrome. Arch Gen Psychiatry 56:940945. Medline

Nery S, Fishell G, Corbin JG (2002) The caudal ganglionic eminence is a source of distinct cortical and subcortical cell populations. Nat Neurosci 5:1279-1287. CrossRef Medline

Niklasson L, Rasmussen P, Oskarsdóttir S, Gillberg C (2009) Autism, ADHD, mental retardation and behavior problems in 100 individuals with 22q11 deletion syndrome. Res Dev Disabil 30:763-773. CrossRef Medline

Polleux F, Ghosh A (2002) The slice overlay assay: a versatile tool to study the influence of extracellular signals on neuronal development. Sci STKE 2002:p19-pl9. Medline

Polleux F, Whitford KL, Dijkhuizen PA, Vitalis T, Ghosh A (2002) Control of cortical interneuron migration by neurotrophins and PI3-kinase signaling. Development 129:3147-3160. Medline

Powell EM, Mars WM, Levitt P (2001) Hepatocyte growth factor/scatter factor is a motogen for interneurons migrating from the ventral to dorsal telencephalon. Neuron 30:79-89. CrossRef Medline

Pozas E, Ibáñez CF (2005) GDNF and GFRalphal promote differentiation and tangential migration of cortical GABAergic neurons. Neuron 45:701713. CrossRef Medline

Qu C, Li W, Shao Q, Dwyer T, Huang H, Yang T, Liu G (2013) c-Jun $\mathrm{N}$-terminal kinase 1 (JNK1) is required for coordination of netrin signaling in axon guidance. J Biol Chem 288:1883-1895. CrossRef Medline

Sabapathy K, Jochum W, Hochedlinger K, Chang L, Karin M, Wagner EF (1999) Defective neural tube morphogenesis and altered apoptosis in the absence of both JNK1 and JNK2. Mech Dev 89:115-124. CrossRef Medline

Sánchez-Alcañiz JA, Haege S, Mueller W, Pla R, Mackay F, Schulz S, LópezBendito G, Stumm R, Marín O (2011) Cxcr7 controls neuronal migration by regulating chemokine responsiveness. Neuron 69:77-90. CrossRef Medline

Stenman J, Toresson H, Campbell K (2003) Identification of two distinct progenitor populations in the lateral ganglionic eminence: implications for striatal and olfactory bulb neurogenesis. J Neurosci 23:167-174. Medline

Stumm RK, Zhou C, Ara T, Lazarini F, Dubois-Dalcq M, Nagasawa T, Höllt V, Schulz S (2003) CXCR4 regulates interneuron migration in the developing neocortex. J Neurosci 23:5123-5130. Medline

Stumm R, Kolodziej A, Schulz S, Kohtz JD, Höllt V (2007) Patterns of SDF-1 alpha and SDF-1 gamma mRNAs, migration pathways, and phenotypes of CXCR4-expressing neurons in the developing rat telencephalon. J Comp Neurol 502:382-399. CrossRef Medline

Sussel L, Marin O, Kimura S, Rubenstein JL (1999) Loss of Nkx2.1 homeobox gene function results in a ventral to dorsal molecular respecification within the basal telencephalon: evidence for a transformation of the pallidum into the striatum. Development 126:3359-3370. Medline

Tahirovic S, Hellal F, Neukirchen D, Hindges R, Garvalov BK, Flynn KC, Stradal TE, Chrostek-Grashoff A, Brakebusch C, Bradke F (2010) Rac1 regulates neuronal polarization through the WAVE complex. J Neurosci 30:6930-6943. CrossRef Medline

Tiveron MC, Rossel M, Moepps B, Zhang YL, Seidenfaden R, Favor J, König N, Cremer H (2006) Molecular interaction between projection neuron precursors and invading interneurons via stromal-derived factor 1 (CXCL12)/CXCR4 signaling in the cortical subventricular zone/intermediate zone. J Neurosci 26:13273-13278. CrossRef Medline

Tivodar S, Kalemaki K, Kounoupa Z, Vidaki M, Theodorakis K, Denaxa M, Kessaris N, Decurtis I, Pachnis V, Karagogeos D (2014) Rac-GTPases regulate microtubule stability and axon growth of cortical GABAergic interneurons. Cereb Cortex. Advance online publication. doi:10.1093/ cercor/bhu037. CrossRef

Toritsuka M, Kimoto S, Muraki K, Landek-Salgado MA, Yoshida A, Yamamoto N, Horiuchi Y, Hiyama H, Tajinda K, Keni N, Illingworth E, Iwamoto T, Kishimoto T, Sawa A, Tanigaki K (2013) Deficits in microRNA-mediated Cxcr4/Cxcl12 signaling in neurodevelopmental deficits in a 22q11 deletion syndrome mouse model. Proc Natl Acad Sci U S A 110:17552-17557. CrossRef Medline

Tucker ES, Polleux F, LaMantia AS (2006) Position and time specify the migration of a pioneering population of olfactory bulb interneurons. Dev Biol 297:387-401. CrossRef Medline

Wang X, Nadarajah B, Robinson AC, McColl BW, Jin JW, Dajas-Bailador F, Boot-Handford RP, Tournier C (2007) Targeted deletion of the mitogen-activated protein kinase kinase 4 gene in the nervous system causes severe brain developmental defects and premature death. Mol Cell Biol 27:7935-7946. CrossRef Medline

Wang Y, Li G, Stanco A, Long JE, Crawford D, Potter GB, Pleasure SJ, Behrens T, Rubenstein JL (2011) CXCR4 and CXCR7 have distinct functions in regulating interneuron migration. Neuron 69:61-76. CrossRef Medline

Westerlund N, Zdrojewska J, Padzik A, Komulainen E, Björkblom B, Rannikko E, Tararuk T, Garcia-Frigola C, Sandholm J, Nguyen L, Kallunki T, Courtney MJ, Coffey ET (2011) Phosphorylation of SCG10/stathmin-2 determines multipolar stage exit and neuronal migration rate. Nat Neurosci 14:305-313. CrossRef Medline

Wichterle H, Turnbull DH, Nery S, Fishell G, Alvarez-Buylla A (2001) In utero fate mapping reveals distinct migratory pathways and fates of neurons born in the mammalian basal forebrain. Development 128:37593771. Medline

Yamasaki T, Kawasaki H, Arakawa S, Shimizu K, Shimizu S, Reiner O, Okano H, Nishina S, Azuma N, Penninger JM, Katada T, Nishina H (2011) Stress-activated protein kinase MKK7 regulates axon elongation in the developing cerebral cortex. J Neurosci 31:16872-16883. CrossRef Medline

Yamasaki T, Kawasaki H, Nishina H (2012) Diverse roles of JNK and MKK pathways in the brain. J Signal Transduct 2012:459265-459265. CrossRef Medline

Yang DD, Conze D, Whitmarsh AJ, Barrett T, Davis RJ, Rincón M, Flavell RA (1998) Differentiation of CD4(+) T cells to Th1 cells requires MAP kinase JNK2. Immunity 9:575-585. CrossRef Medline

Yau HJ, Wang HF, Lai C, Liu FC (2003) Neural development of the neuregulin receptor ErbB4 in the cerebral cortex and the hippocampus: preferential expression by interneurons tangentially migrating from the ganglionic eminences. Cereb Cortex 13:252-264. CrossRef Medline 